Hydroécol. Appl. (1994) Tome 6 Vol 1-2, pp. 87-114

\title{
Evolution saisonnière des éléments minéraux nutritifs $(P, N, S i)$ et de l'oxygène dissous dans le lac de Pareloup
}

\section{Seasonal evolution of dissolved inorganic nutrients $(P, N, S i)$ and oxygen in Pareloup lake}

\author{
L. Labroue*, J. Capblancq*, M.J. Salençon ${ }^{\star *}$, J.N. Tourenq* \\ and C. Mur* \\ * Laboratoire d'Hydrobiologie, U.R.A. 695 C.N.R.S., Université Paul Sabatier, 118 Route de \\ Narbonne, F-31062 Toulouse Cedex, France. \\ ** Electricité de France, Direction des Etudes et Recherches. Département Environnement. \\ 6 Quai Watier, F-78401 Chatou Cedex, France.
}

Résumé. - Le contrôle exercé par les stocks de phosphore et de silice disponibles sur le cycle de développement du phytoplancton dans le lac de Pareloup est mis en évidence par l'évolution comparée des concentrations de chlorophylle, de phosphore, de nitrate et de silice dans la zone pélagique de ce lac au cours de deux cycles annuels. La sédimentation des diatomées qui se développent au printemps contribue pour une large part au transfert de $\mathrm{P}$ et de Si dans les sédiments. Les flux d'éléments relargués à partir des sédiments (charge interne), évalués par des mesures en chambres benthiques, sont ponctuellemęnt élevés mais interviennent probablement peu dans les bilans en comparaison des apports par les eaux pompées dans le réservoir de Bage. L'influence des apports de Bage apparaît de façon particulièrement nette lorsqu'on considère la demande hypolimnique en oxygène dissous. Les concentrations réelles de $P$ total dans l'eau de Pareloup sont cependant bien inférieures à ce que laisseraient prévoir ces apports, ce qui suggère une influence favorable qui reste à préciser de la gestion des eaux.

Mots-clés. - Azote, cycle des nutriments, oxygène dissous, phosphore, silice.

Abstract. - A comparison of variations in chlorophyll, phosphorus, nitrate and silica concentrations in the pelagic zone of Pareloup reservoir over two annual cycles suggests that phosphorus and silica availability probably control the seasonal phytoplankton biomass cycle. Sinking of the spring diatom bloom is largely responsible for the transfer 
of $\mathrm{P}$ and Si from water to sediments. Fluxes of nutrients released from the sediments (internal load), as measured by the benthic chambers method, are occasionally high but probably of minor importance in the budgets, as compared with external inputs from the Bage reservoir. The impact of these inputs is clearly illustrated by the local hypolimnetic oxygen demand. The real total phosphorus concentrations in the water column at Pareloup remain well below those one might predict on the basis of inputs, suggesting a positive influence of the hydraulic management mode which remains to be identified.

Key words. - Nitrogen, nutrient cycle, dissolved oxygen, phosphorus, silica.

\section{INTRODUCTION}

Situé au sud-ouest du Massif Central à une altitude de 800 mètres, le réservoir de Pareloup a un volume de $169 \mathrm{hm}^{3}$ pour une superficie de 1260 ha à sa cote maximale. Sa profondeur moyenne est de 12,5 mètres, la profondeur maximale au niveau du barrage est de 40 mètres.

Le réservoir est alimenté par les apports naturels d'un bassin versant de $120 \mathrm{~km}^{2}$ (bassin du Vioulou) et par des eaux pompées dans le réservoir de Bage $\left(4 \mathrm{hm}^{3}\right)$ qui est lui même alimenté par la retenue de Pont du Salars $\left(20 \mathrm{hm}^{3}\right)$. Les eaux pompées dans Bage sont injectées avec un débit maximum de $14 \mathrm{~m}^{3} \cdot \mathrm{s}^{-1}$ dans le fond du réservoir de Pareloup au pied du barrage. L'eau emmagasinée est utilisée en période de forte demande, principalement l'hiver, pour produire de l'électricité. Le temps de rétention moyen de l'eau dans le réservoir de Pareloup est de 300 jours; la variation du niveau de l'eau dans la retenue est au maximum de \pm 10 mètres.

Le régime thermique du lac de $\mathrm{Pa}$ reloup est du type monomictique chaud à dimictique lors des hivers rigoureux (fig. 1). Il se caractérise par une longue période d'isothermie (fin septembre-début juin), avec une stratification thermique inverse certaines années, à laquelle succède une phase de stratification thermique d'une durée de trois mois et demi. Le gradient thermique qui se forme progressivement à partir du début du mois de juin atteint son maximum en août.

Issues d'un bassin versant essentiellement cristallin, les eaux de Pareloup sont faiblement minéralisées (tableau I). La conductivité à $20^{\circ} \mathrm{C}$ est de $89,3 \mu \mathrm{S}$ en moyenne. Le $\mathrm{pH}$ moyen est de 7,3 avec des valeurs extrêmes de 6,75 et de 7,88 .

Les résultats présentés ici concernent l'évolution des principaux éléments biogènes (phosphore, azote, silice, oxygène) au cours des années 1986 et 1987.

\section{MATÉRIEL ET MÉTHODES}

Les températures et les concentrations en oxygène ont été mesurées " in situ» avec une sonde immergeable YSI (thermosonde et cellule polarographique). 

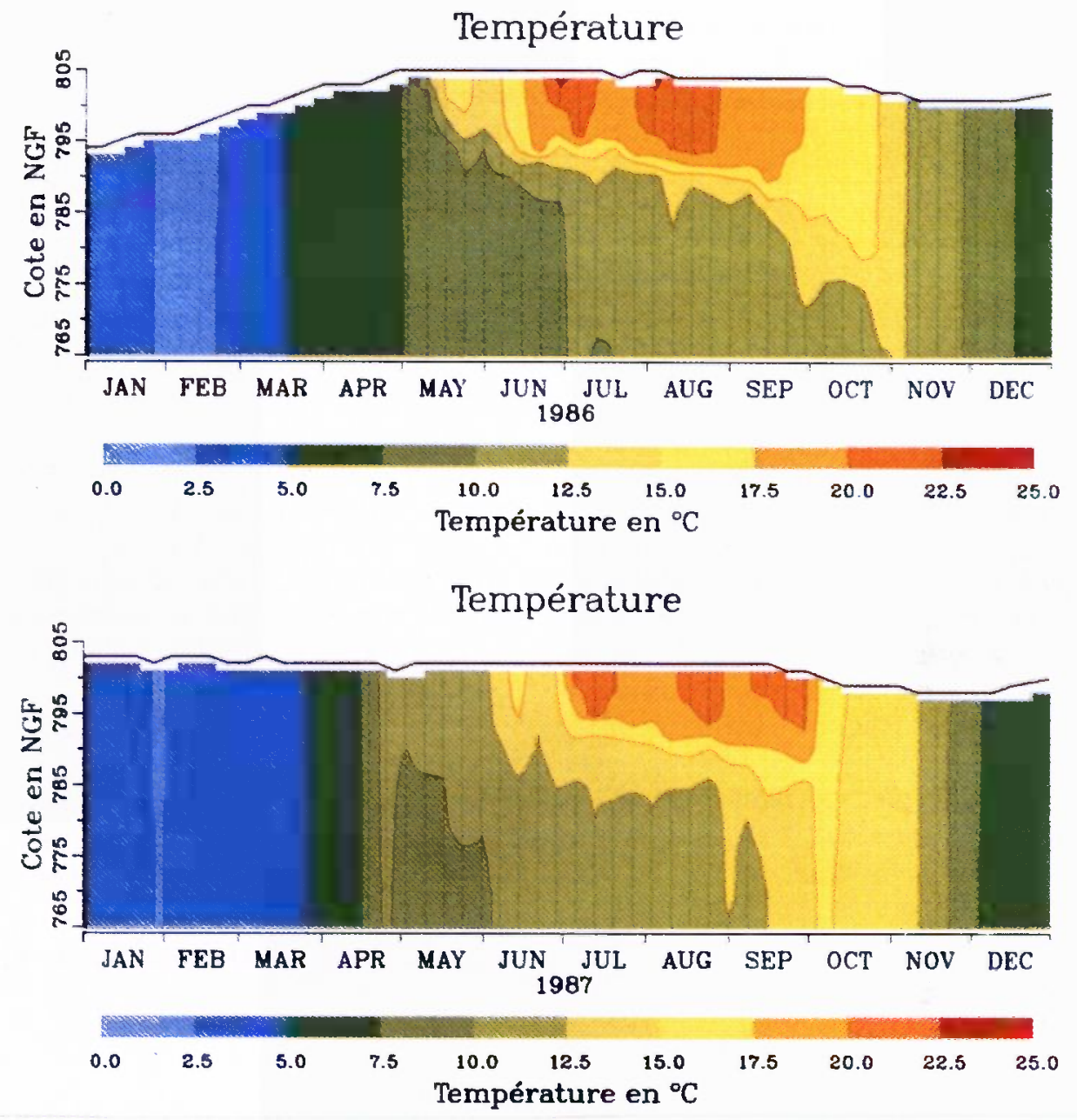

Fig. 1. - Evolution saisonnière de la température dans la colonne d'eau du lac de Pareloup en 1986 et 1987.

Fig. 1. - Temperature isopleths for Pareloup lake, 1986 and 1987.

Les analyses chimiques ont été effectuées sur des échantillons d'eau prélevés à la bouteille VAN DORN, transportés dans des glacières et conservés un minimum de temps dans des réfrigérateurs.
Les nitrates ont été analysés par dosage colorimétrique (réactif de Griess) des nitrites formés par réduction sur colonne de Cadmium.

Le phosphore a été dosé par colorimétrie du complexe de phospho- 
Tableau I. - Concentrations moyennes des anions et des cations majeurs dans l'eau du lac de Pareloup.

Table I. - Ionic composition of the water of Pareloup.

\begin{tabular}{|lcc|lcc|}
\hline & meq. $\Gamma^{-1}$ & anions $\%$ & & meq. ${ }^{-1}$ & cations \% \\
\hline $\mathrm{HCO}_{3}^{-}$ & 0,450 & 57,0 & $\mathrm{Ca}^{+2}$ & 0,354 & 43,0 \\
$\mathrm{Cl}^{-}$ & 0,141 & 17,8 & $\mathrm{Mq}^{+2}$ & 0,238 & 29,0 \\
$\mathrm{SO}_{4}^{-2}$ & 0,079 & 10,0 & $\mathrm{Na}^{+}$ & 0,169 & 20,5 \\
$\mathrm{NO}_{3}^{-}$ & 0,120 & 15,2 & $\mathrm{~K}^{+}$ & 0,061 & 7,5 \\
\hline Total & 0,790 & 100 & Total & 0,822 & 100 \\
\hline
\end{tabular}

molybdate et de vert de malachite en milieu acide (Motomizu et al., 1983). Le dosage pratiqué directement sur de l'eau filtrée à $0,45 \mu \mathrm{m}$ concerne le phosphore réactif soluble (PRS), souvent assimilé à tort au phosphore de l'ion $\mathrm{PO}_{4}$ libre. Le dosage pratiqué sur l'eau filtrée mais minéralisée auparavant (autoclavage en mileu acide additionné de persulfate) fournit le phosphore dissous total. La même opération réalisée sur l'eau brute permet de mesurer le phosphore total. Le phosphore particulaire peut être obtenu par différence ou, plus précisément, par dosage du matériel retenu sur le filtre après minéralisation.

Le carbone et l'azote particulaires ont été dosés après combustion à $900^{\circ} \mathrm{C}$ en présence d'oxyde de cuivre du matériel retenu sur les filtres, le $\mathrm{CO}_{2}$ et le $\mathrm{N}_{2}$ émis, étant analysés par chromatographie en phase gazeuse (Dalger, 1982)

La silice a été dosée par colorimétrie du complexe silico-molybdique réduit.

Les échanges sédiments-eau ont été étudiés dans des cloches benthi- ques de $20 \mathrm{~cm}$ de diamètre et de $12 \mathrm{I}$. de contenance. La circulation dans les cloches était assurée par des tubes en polyéthylène et une pompe péristaltique, les prélèvements étant assurés au niveau d'un $T$ muni d'un bouchon à jupe rabattable.

L'eau interstitielle des sédiments a été obtenue après une centrifugation rapide des divers horizons de carottes dans des tubes pleins et bouchés pour réduire l'oxydation. Le surnageant a été filtré à $0,22 \mu \mathrm{m}$ et conservé dans des tubes pleins et bouchés.

\section{LE PHOSPHORE}

\section{Évolution du phosphore dans la colonne d'eau}

Les eaux de Pareloup contiennent en moyenne $15 \mu \mathrm{g} \cdot \mathrm{I}^{-1}$ de phosphore total (fig. 2). Les concentrations sont légèrement plus élevées en période de brassage hivernal et printanier (20 à $25 \mu \mathrm{g} \cdot \mathrm{f}^{-1}$ ) qui correspond à l'essentiel des apports de $P$ dans la retenue par les affluents et les eaux pompées 

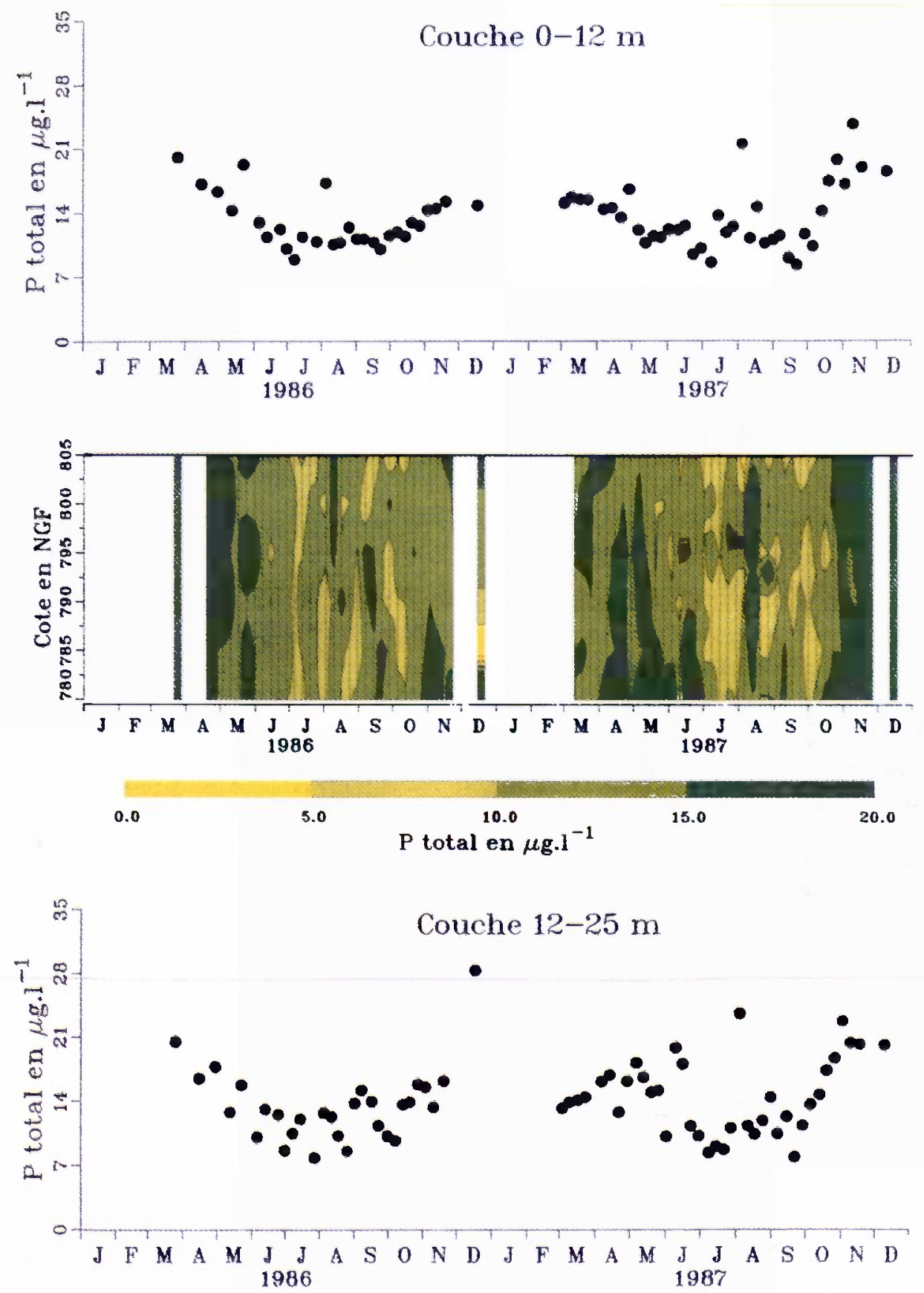

Fig. 2. - Evolution saisonnière de la concentration en Phosphore total dans la colonne d'eau du lac de Pareloup en 1986 et 1987: concentration moyenne dans les couches 0-12 m (en haut), $12-25 \mathrm{~m}$ (en bas) et courbes d'isoconcentration (au centre).

Fig. 2. - Seasonal evolution of the total phosphorus concentration in the water column at Pareloup in 1986 and 1987: mean concentration in layers 0-12 $\mathrm{m}$ (top) and 12-25 $\mathrm{m}$ (bottom) and depth-time distribution (center). 
dans le réservoir de Bage (Sivadier et al., 1994). Les teneurs minimales de $\mathrm{P}$ total $\left(8 \mathrm{à} 10 \mu \mathrm{g} \cdot \mathrm{l}^{-1}\right)$ s'observent dans l'épilimnion en période de stratification estivale.

Les concentrations moyennes de $\mathrm{N}-\mathrm{NO}_{3}\left(1450 \mu \mathrm{g} \cdot \mathrm{l}^{-1}\right)$ et de $\mathrm{P}$ total $\left(15 \mu \mathrm{g} \cdot \mathrm{l}^{-1}\right)$ sont dans un rapport $\mathrm{N}: \mathrm{P}=100$ indiquant que la production de phytoplancton est vraissemblablement contrôlée par le phosphore disponible. L'analyse élémentaire des matières particulaires (fraction 0,45$200 \mu \mathrm{m}$ ) révèle cependant une relative constance du rapport N/P dont la valeur moyenne $(8,4$ en masse; 18,6 en atomes) correspond à celle de populations d'algues proches de leur taux optimal de croissance (Healey \& Hendzel, 1979; Goldman 1980). L'in- terférence du matériel détritique se traduit par des rapports $\mathrm{C}: \mathrm{Chl}, \mathrm{N}: \mathrm{Chl}$ et $\mathrm{P}: \mathrm{Chl}$ de l'ordre de $200 ; 20$ et 2 respectivement lorsque la biomasse de phytoplancton est faible; lorsque le phytoplancton constitue une part importante des matières en suspension

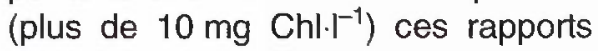
sont de l'ordre de $60 ; 7$ et 0,8 (fig. 3 ).

La plus grande partie du P-total (61\% en moyenne) est associée aux particules retenues par des filtres d'une porosité de $0,45 \mu \mathrm{m}$. La fraction inférieure à $0,45 \mu \mathrm{m}$ correspond à des teneurs de $P$ total dissous généralement comprises entre 2 et $8 \mu \mathrm{g} \cdot \mathrm{I}^{-1}$ (fig. 4). Celui ci est composé pour $52 \%$ en moyenne de "phosphore réactif soluble" (PRS) dont le dosage chimique avec des réactifs acides

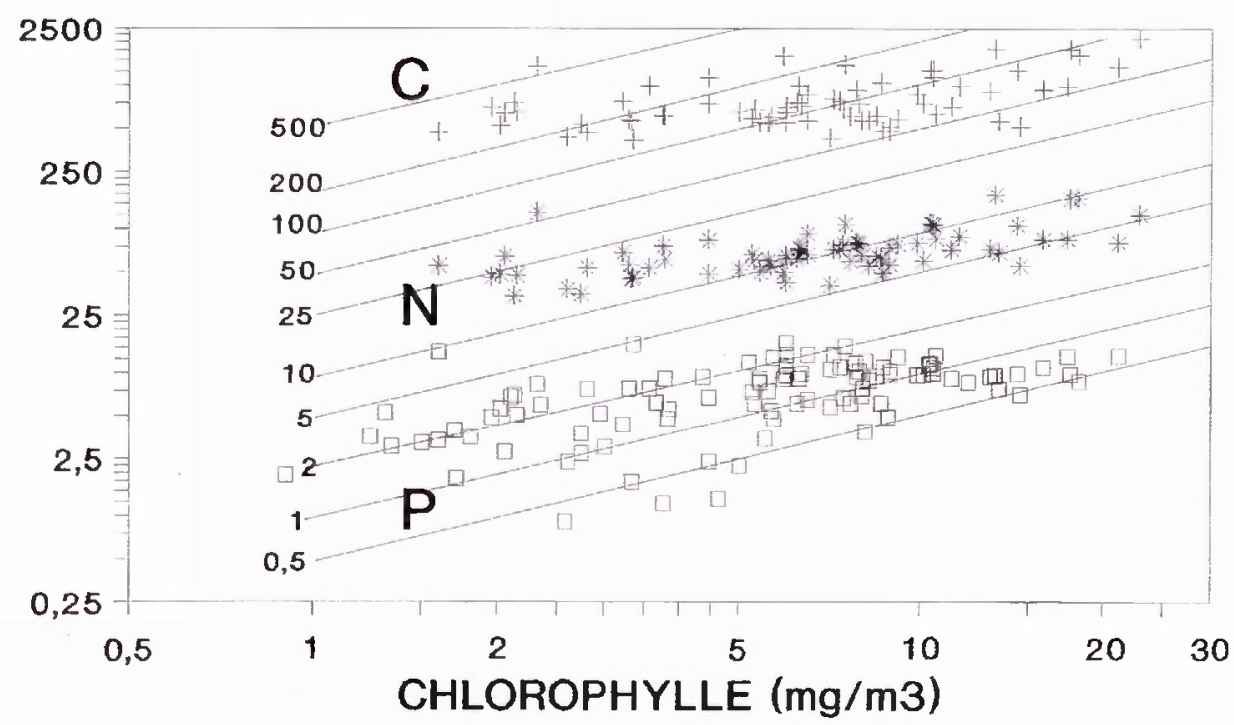

Fig. 3. - Relations Chlorophylle a - Carbone, Azote, Phosphore particulaires des matières en suspension au lac de Pareloup.

Fig. 3. - Relationships between chlorophyll $a$ and the carbon, nitrogen and phosphorus content in suspended solids in Pareloup. 

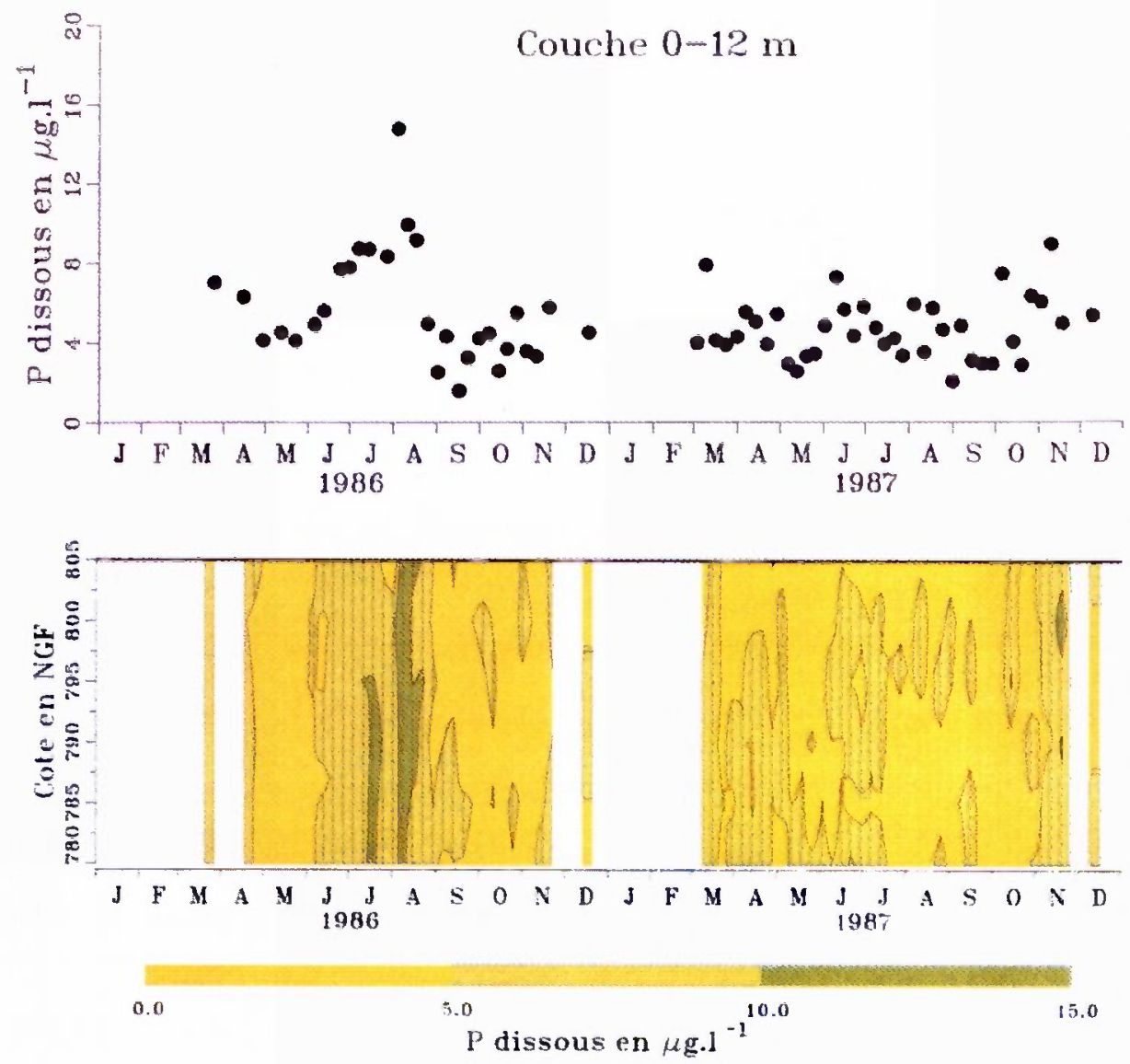

15.0

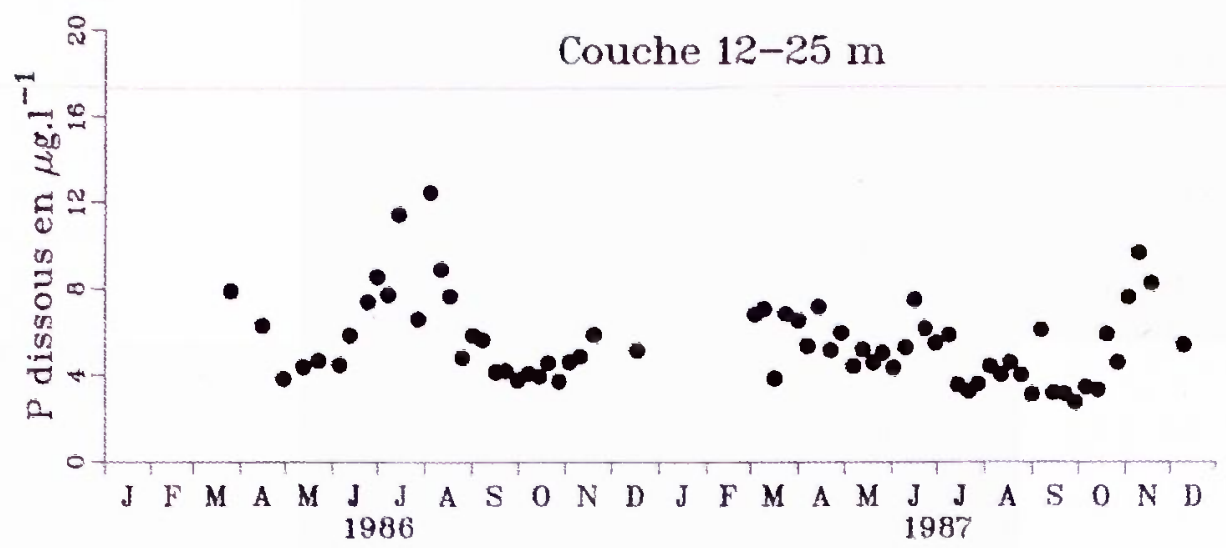

Fig. 4. - Evolution saisonnière de la concentration en Phosphore dissous dans la colonne d'eau du lac de Pareloup en 1986 et 1887 : concentration moyenne dans les couches $0-12 \mathrm{~m}$ (en haut), $12-25 \mathrm{~m}$ (en bas) et courbes d'isoconcentration (au centre).

Fig. 4. - Seasonal evolution of the dissolved phosphorus concentration in the water column at Pareloup in 1986 and 1987: mean concentration in layers 0-12 $\mathrm{m}$ (top) and 12-25 m (bottom) and depth-time distribution (center). 
donne des valeurs supérieures aux quantités de $\mathrm{P}_{-} \mathrm{PO}_{4}$ réellement présentes (Rigler, 1968). Les concentrations de PRS sont de $2,7 \mu \mathrm{g} \cdot \mathrm{I}^{-1}$ en moyenne et varient entre 0,1 et $12 \mu \mathrm{g} \cdot \mathrm{I}^{-1}$, (fig. 5) les plus fortes teneurs correspondant au minimum estival de biomasse de phytoplancton (fig. 6). Ces fluctuations des teneurs en PRS résultent d'une dynamique complexe dans laquelle interviennent des échanges permanents et rapides au sein de la masse d'eau entre le $\mathrm{P}_{-} \mathrm{PO}_{4}$ dissous, la fraction colloïdale et les particules inertes et vivantes du seston (Lean, 1973), la régénération de $\mathrm{PO}_{4}$ par le zooplancton et les apports variables de $\mathrm{P}$ dans la zone pélagique dépendant de l'hydrodynamique. En conséquence, les concentrations de PRS sont à considérer comme une valeur transitoire et non comme une mesure de la quantité de phosphore biodisponible.

Les bilans entrée-sortie de phosphore dans le réservoir de Pareloup indiquent que les $3 / 4$ du $\mathrm{P}$ total importé sont stockés dans les sédiments. La sédimentation des diatomées qui se développent au printemps contribue à ce transfert de phosphore vers les sédiments pour une part que l'on peut évaluer ainsi :

- les dosages de chlorophylle contenue dans les matières recueillies dans des pièges à sédiments entre le 10 mars et le 15 juin correspondent à $705 \mathrm{mg} \cdot \mathrm{m}^{-2}$ en 1986 et $420 \mathrm{mg} \cdot \mathrm{m}^{-2}$ en 1987. Les relations P-Chla du matériel en suspension pendant cette période indiquant un rapport $\mathrm{P} / \mathrm{Chla}$ moyen de l'ordre de 0,85 (fig: 3 ), la quantité de chlorophylle sédimentée équivaut à $600 \mathrm{mg} \mathrm{P} \cdot \mathrm{m}^{-2}$ en 1986 et $360 \mathrm{mg} \mathrm{P} \cdot \mathrm{m}^{-2}$ en 1987. Comparées avec les quantités de phosphore total recueillies pendant les mêmes périodes dans les pièges à sédiments (585 $\mathrm{mg} \cdot \mathrm{m}^{-2}$ en 1986; $655 \mathrm{mg} \cdot \mathrm{m}^{-2}$ en 1987) ces valeurs permettent d'estimer que $66 \%$ à $100 \%$ du flux de $P$ vers les sédiments pendant la période printanière sont attribuables à la sédimentation des diatomées.

- les modèles de production photosynthétique permettent d'estimer à $23 \mathrm{~g} \mathrm{C} \cdot \mathrm{m}^{-2}$ en 1986 et $24 \mathrm{~g} \mathrm{C} \cdot \mathrm{m}^{-2}$ en 1987 la production brute du phytoplancton entre le 10 mars et le 15 juin. En admettant que $35 \%$ du carbone assimilé sont consommés par respiration, la production primaire nette est de l'ordre de $15 \mathrm{~g} \mathrm{C} \cdot \mathrm{m}^{-2}$ ce qui correspond, pour un rapport $C / P=40$, à $375 \mathrm{mg} \mathrm{P} \cdot \mathrm{m}^{-2}$ mobilisés par le phytoplancton. La sédimentation de cette biomasse produite représente donc $64 \%$ (1986) et $57 \%(1987)$ du P recueilli dans les pièges à sédiments entre le 10 mars et le 15 juin.

\section{Les échanges sédiments-eau}

Les sédiments du lac fonctionnent comme un piège à phosphore. Ils reçoivent du phosphore particulaire et adsorbent, éventuellement, du phosphore dissous. Le phosphore particulaire allochtone est essentiellement détritique, le phosphore autochtone est du matériel biogène lacustre, issu des algues en particulier.

Les échanges sédiment-eau ont été évalués in situ par le système des cloches benthiques (fig. 7 et 8 ). 

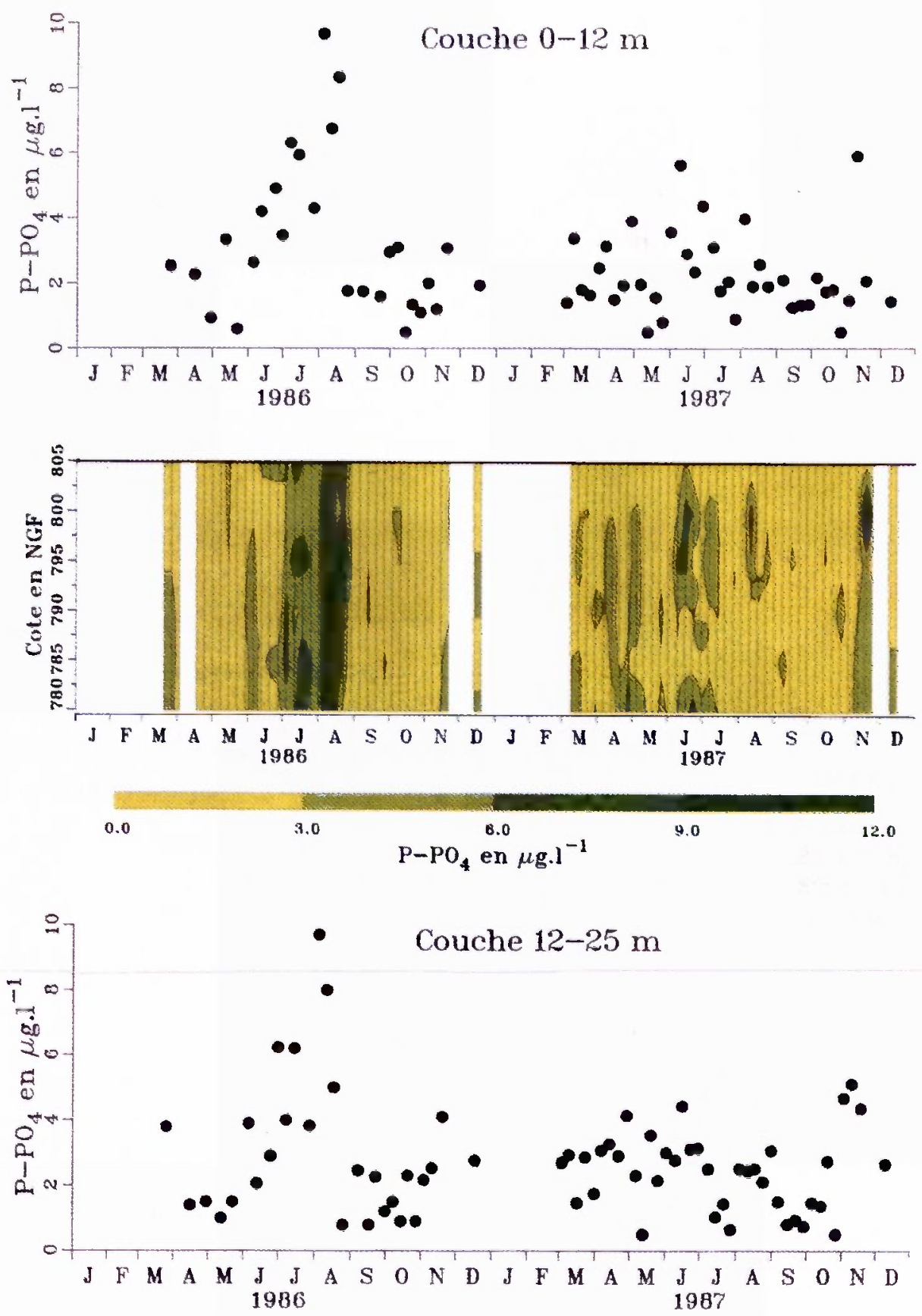

Fig. 5. - Evolution saisonnière de la concentration en Phosphore $\mathrm{PO}_{4}$ (P.R.S.) dans la colonne d'eau du lac de Pareloup en 1986 et 1987: concentration moyenne dans les couches 0-12 m (en haut), $12-25 \mathrm{~m}$ (en bas) et courbes d'isoconcentration (au centre).

Fig. 5. - Seasonal evolution of the phosphorus $\mathrm{PO}_{4}$ (S.R.P.) concentration in the water column at Pareloup in 1986 and 1987: mean concentration in layers 0-12 $\mathrm{m}$ (top) and 12-25 m (bottom) and depth-time distribution (center). 

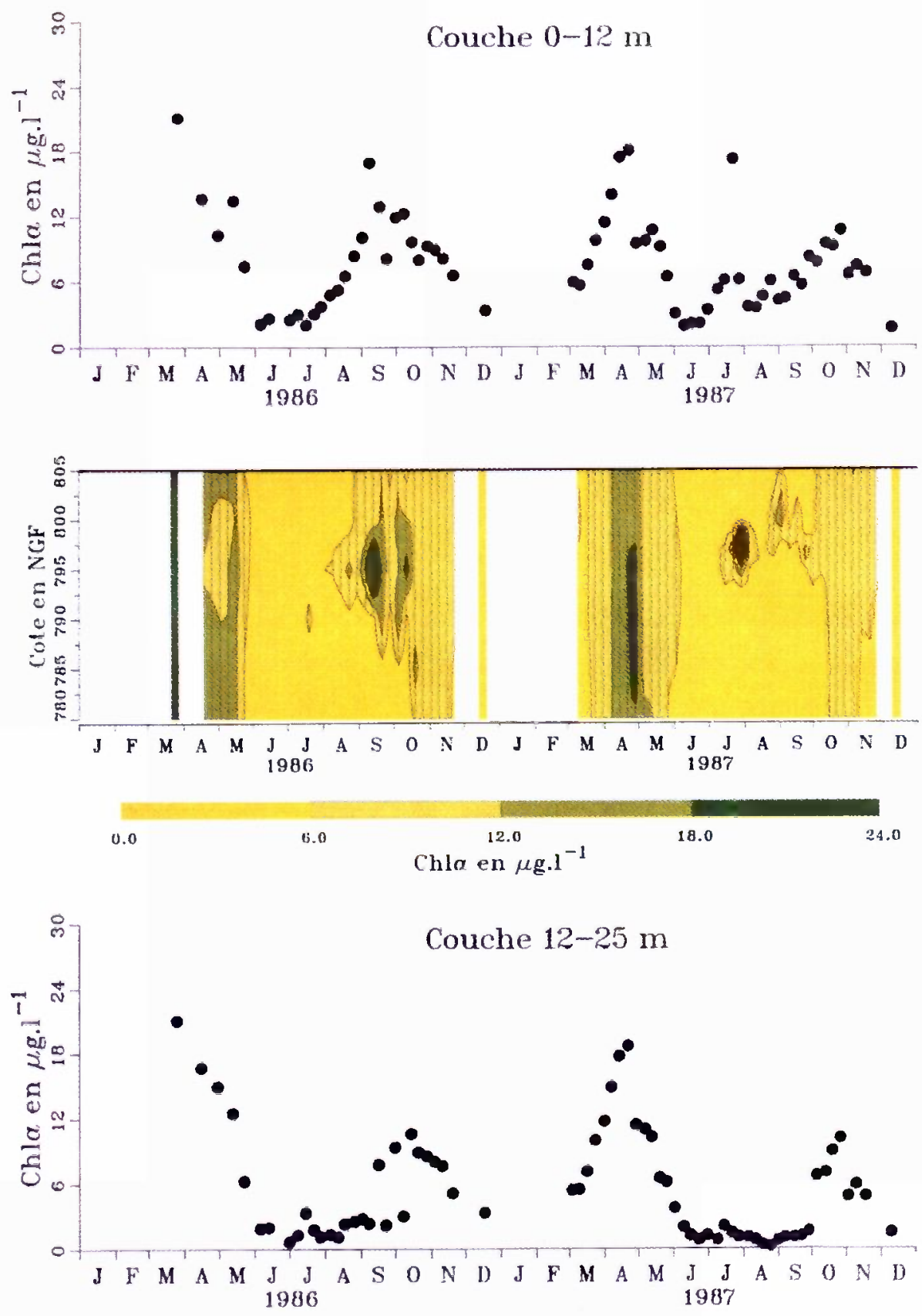

Fig. 6. - Evolution saisonnière de la concentration en chlorophylle dans la colonne d'eau du lac de Pareloup en 1986 et 1987 : concentration moyenne dans les couches 0-12 m (en haut), 12-25 m (en bas) et courbes d'isoconcentration (au centre).

Fig. 6. - Seasonal evolution of the chlorophyll concentration in the water column at Pareloup in 1986 and 1987: mean concentration in layers 0-12 m (top) and 12-25 m (bottom) and depth-time distribution (center). 

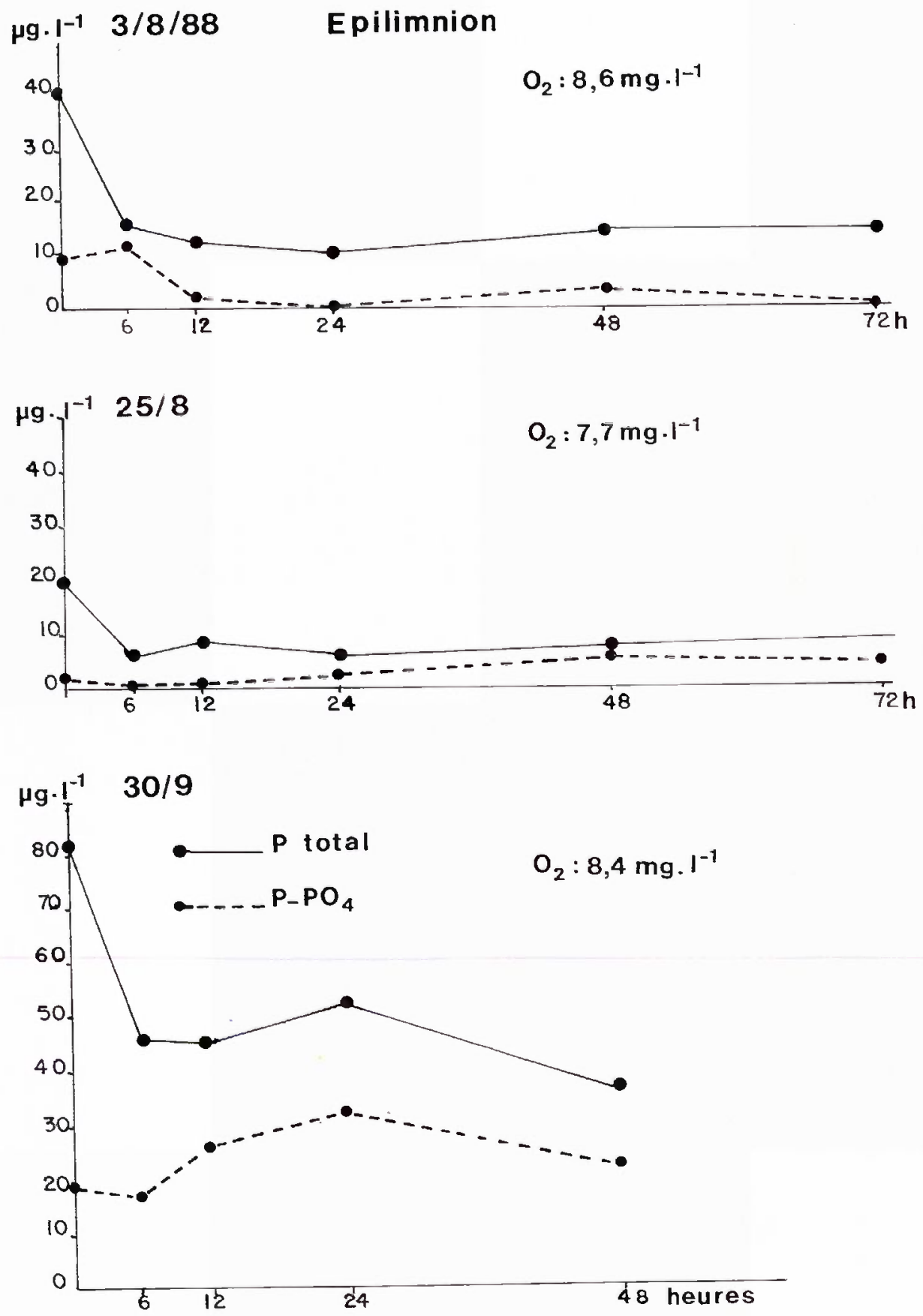

Fig. 7. - Evolution de la concentration en Phosphore total et en P-POH (P.R.S.) dans les cloches benthiques en zone épilimnique au cours de l'été 1988.

Fig. 7. - Evolution of the total phosphorus and $\mathrm{P}_{-} \mathrm{PO}_{4}$ (S.R.P.) concentration in the benthic chambers in the epilimnetic zone in summer 1988. 

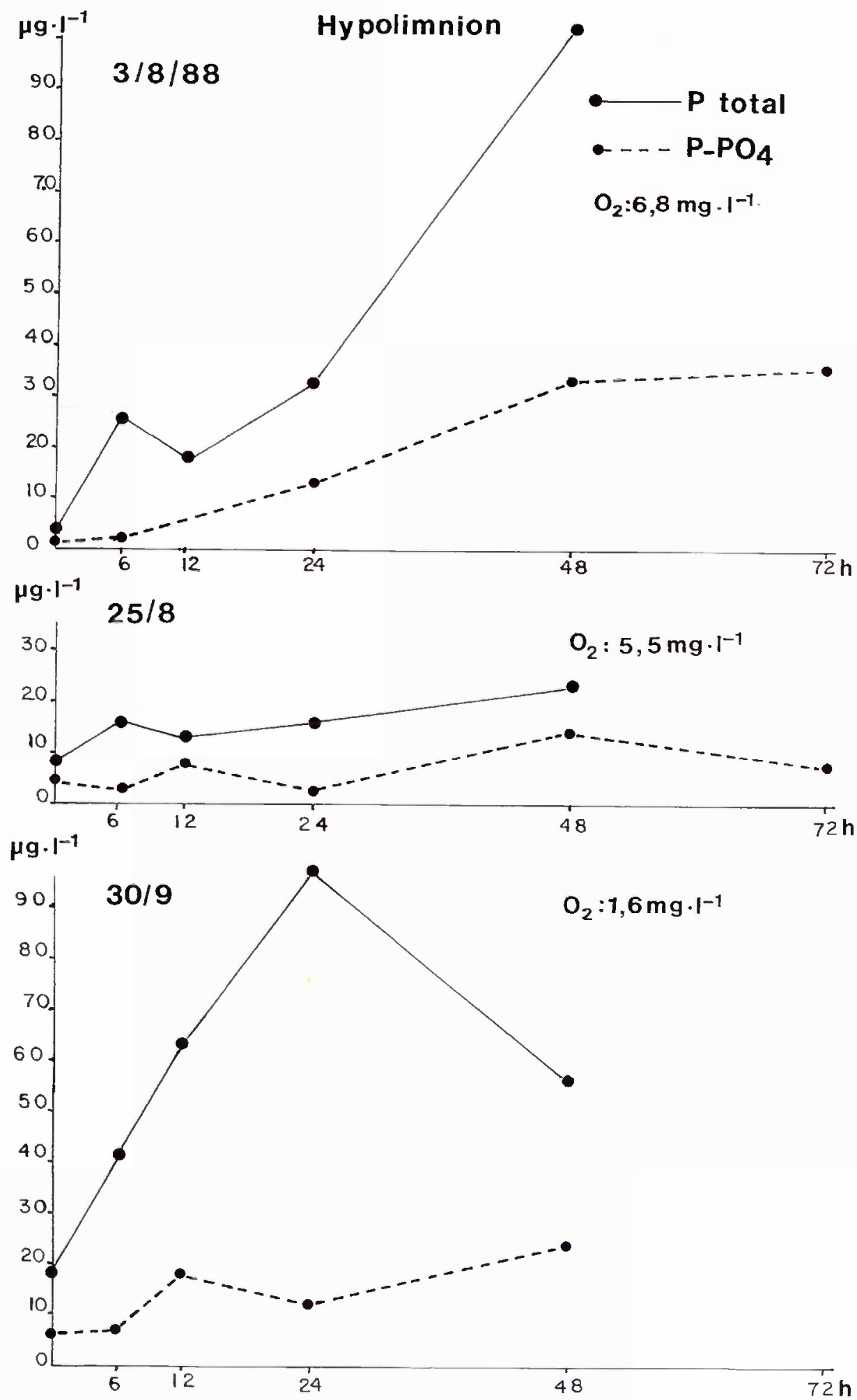

Fig. 8. - Evolution de la concentration en Phosphore total et en P-PO 4 (P.R.S.) dans les cloches benthiques en zone hypolimnique au cours de l'été 1988.

Fig. 8. - Evolution of the total phosphorus and $\mathrm{P}_{-} \mathrm{PO}_{4}$ (S.R.P.) concentration in the benthic chambers in the hypolimnetic zone in summer 1988. 
La zone épilimnique apparaît plutôt fixatrice, avec des concentrations d'équilibre très basses, inférieures à $5 \mu \mathrm{g} \cdot \mathrm{I}^{-1}$. Cette propriété disparaît à la fin septembre, au moment de la destratification, lorsque les sédiments hypolimniques et les apports de Bage se répandent sur la zone épilimnique. Ce phénomène est aussi mis en évidence par l'ammoniaque (cf. le cycle de l'azote). La teneur en phosphore augmente alors brutalement et la concentration d'équilibre du $\mathrm{P}_{-} \mathrm{PO}_{4}$ passe à $30 \mu \mathrm{g} \cdot \mathrm{J}^{-1}$.

Au contraire, la zone hypolimnique produit un flux de phosphore ("charge interne") qui augmente, de manière classique avec la désoxygénation du milieu. Ce flux peut paraître important si l'on considère le phosphore total $\left(28 \mathrm{mg} \cdot \mathrm{m}^{-2} \cdot \mathrm{j}^{-1}\right)$ mais il n'est pas possible de savoir si l'augmentation des concentrations de $\mathrm{P}$ total dans les cloches résulte d'une remise en suspension discrète au moment du prélèvement ou d'une fixation par le seston du $\mathrm{P}-\mathrm{PO}_{4}$ relargué par les sédiments. Le flux de $\mathrm{P}_{-} \mathrm{PO}_{4}$ (PRS) est de l'ordre de $4 \mathrm{mg} \cdot \mathrm{m}^{-2} \cdot \mathrm{j}^{-1}$. L'équilibre avec le $P$ particulaire se situe au delà de $20 \mu \mathrm{g} \cdot \mathrm{I}^{-1}$ ce qui correspond aux teneurs de la période de brassage ou aux apports de Bage.

La concentration des eaux interstitielles en phosphore soluble (centrifugation suivie de filtration à $0,2 \mu \mathrm{m}$ ) varie de 0,6 à $0,8 \mathrm{mg} \cdot \mathrm{I}^{-1}$ (fig. 9 ), ce qui correspond au statut de lac mésotrophe. Si l'on utilise la loi de Fick pour calculer un flux (Berner, 1980) à partir du gradient de concentration et d'un coefficient de diffusivité calculé
PS EAU INTERSTITIELLE

DE SEDIMENTS HYPOLIMNIOUES

$31 / 8 / 88$

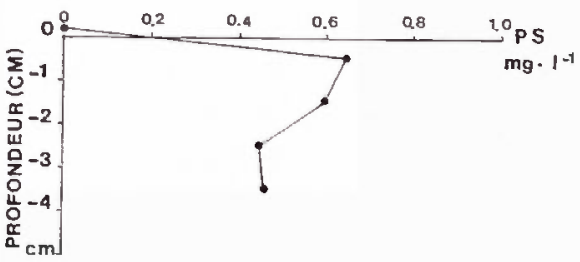

$6 / 9 / 88$

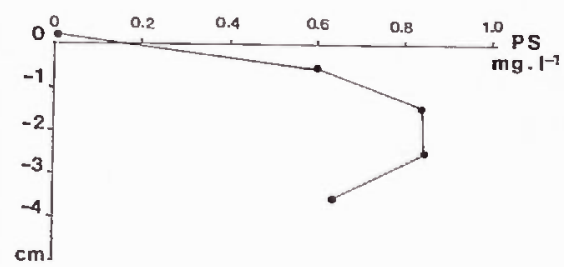

Fig. 9. - Profils de concentration en Phosphore soluble dans l'eau interstitielle des sédiments de la zone hypolimnique du lac de Pareloup au cours de l'été 1988.

Fig. 9. - Profiles of soluble phosphorus concentrations in the interstitial water in the sediments of the hypolimnetic zone at Pareloup in summer 1988.

Ds $=4,43 \cdot 10^{-6} \mathrm{~cm}^{-2} \cdot \mathrm{s}^{-1}$, on obtient un flux de $4,5 \mathrm{mg} \cdot \mathrm{m}^{-2} \cdot \mathrm{j}^{-1}$, proche de celui mesuré dans les cloches benthiques. II est aussi du même ordre de grandeur que la charge externe $\left(4,9 \mathrm{mg} \cdot \mathrm{m}^{-2} \cdot \mathrm{j}^{-1}\right)$ mais notre calcul ne s'applique qu'à la zone hypolimnique et pendant la période estivale alors que la charge externe s'applique à toute la surface du lac et représente une moyenne basée sur l'année. II serait beaucoup plus efficace d'agir sur les apports externes, de Bage en particulier, plutôt que sur les sédiments pour réduire le niveau trophique du lac. 


\section{LA SILICE}

La silice est parfois le facteur limitant de la croissance des Diatomées. C'est, semble-t-il, le cas à la fin du printemps dans le lac de Pareloup.

\section{Évolution de la silice dans la colonne d'eau}

L'évolution saisonnière de la concentration en silice $\left(\mathrm{SiO}_{2}\right)$ du lac durant les années 1986 et 1987 apparaît sur la figure 10. En période hivernale, lorsque la température de l'eau est inférieure à $5^{\circ} \mathrm{C}$, les concentrations atteignent de 55 à $70 \mu \mathrm{M} \cdot \mathrm{I}^{-1}$, ce qui correspond à l'équilibre de saturation de l'illite, un peu supérieur à celui du quartz, pour cette température (Hurd, 1984). Ces valeurs d'équilibre doivent être multipliées par 2 environ pour les températures estivales de l'épilimnion, supérieures à $20^{\circ} \mathrm{C}$. Elles restent bien inférieures à celle de l'équilibre avec la silice des frustules des Diatomées, soit 500 à $1000 \mu \mathrm{M} \cdot \mathrm{I}^{-1}$ (Elgawhary et Lindsay, 1972; Schink et al., 1975).

Les affluents comme le Vioulou montrent des concentrations extrêmement variables, sans périodisme marqué, mais avec une augmentation sensible entre 1986 (valeurs extrêmes entre 50 et $130 \mu \mathrm{M} \cdot \mathrm{l}^{-1}$ ) et 1987 ( 80 à $160 \mu \mathrm{M} \cdot \mathrm{I}^{-1}$ ). Peut-être cela est-il dû au développement du drainage, favorisant la percolation aux dépens du ruissellement.

Un autre élément important des entrées est constitué par l'eau turbinée de la retenue de Bage qui fournit plus de la moitié des débits, en particulier lors de la période hivernale. Cette eau est plus riche en silice, les concentrations allant de 80 à $120 \mu \mathrm{M} \cdot \mathrm{l}^{-1}$ en 1986 , de 100 à $180 \mu \mathrm{M} \cdot \mathrm{l}^{-1}$ en 1987. Elle est à l'origine de la remontée des concentrations en automne et en hiver, l'hypolimnion n'accumulant, par dissolution, que de faibles quantités de silice en été.

La prolifération printanière de Diatomées se traduit par une consommation rapide de la silice dans l'épilimnion. Les concentrations descendent au-dessous de $2 \mu \mathrm{M} \cdot l^{-1}$ comme dans le lac Windermere (Lund et al., 1963 in Paasche, 1980) ou le lac Michigan (Conway et al., 1977). Cette concentration est inférieure aux $6,5 \mu \mathrm{M} \cdot l^{-1}$ à partir desquels la croissance d'Asterionella formosa commence à être limitée (Kilham, 1975, in Paasche, 1980). Il peut être intéressant de rapprocher cette décroissance de la consommation de silice liée à la production primaire des Diatomées pendant la période correspondante.

Au printemps 1986, le bilan dans la colonne d'eau moyenne pour cette période est le suivant :

$$
\begin{gathered}
50 \mu \mathrm{M} \cdot \mathrm{l}^{-1} \times 12 \mathrm{~m}=60 \mu \mathrm{M} \cdot \mathrm{cm}^{-2} \\
\text { ou } 600 \mathrm{mM} \cdot \mathrm{m}^{-2}
\end{gathered}
$$

Au printemps 1987, le même calcul aboutit à $720 \mathrm{mM} \cdot \mathrm{m}^{-2}$

II s'agit d'une estimation, sans doute, par défaut, ne tenant pas compte des entrées-sorties du lac, en particulier des apports de Bage, alors très importants et correspondant à $800 \mathrm{mM} \cdot \mathrm{m}^{-2}$. 

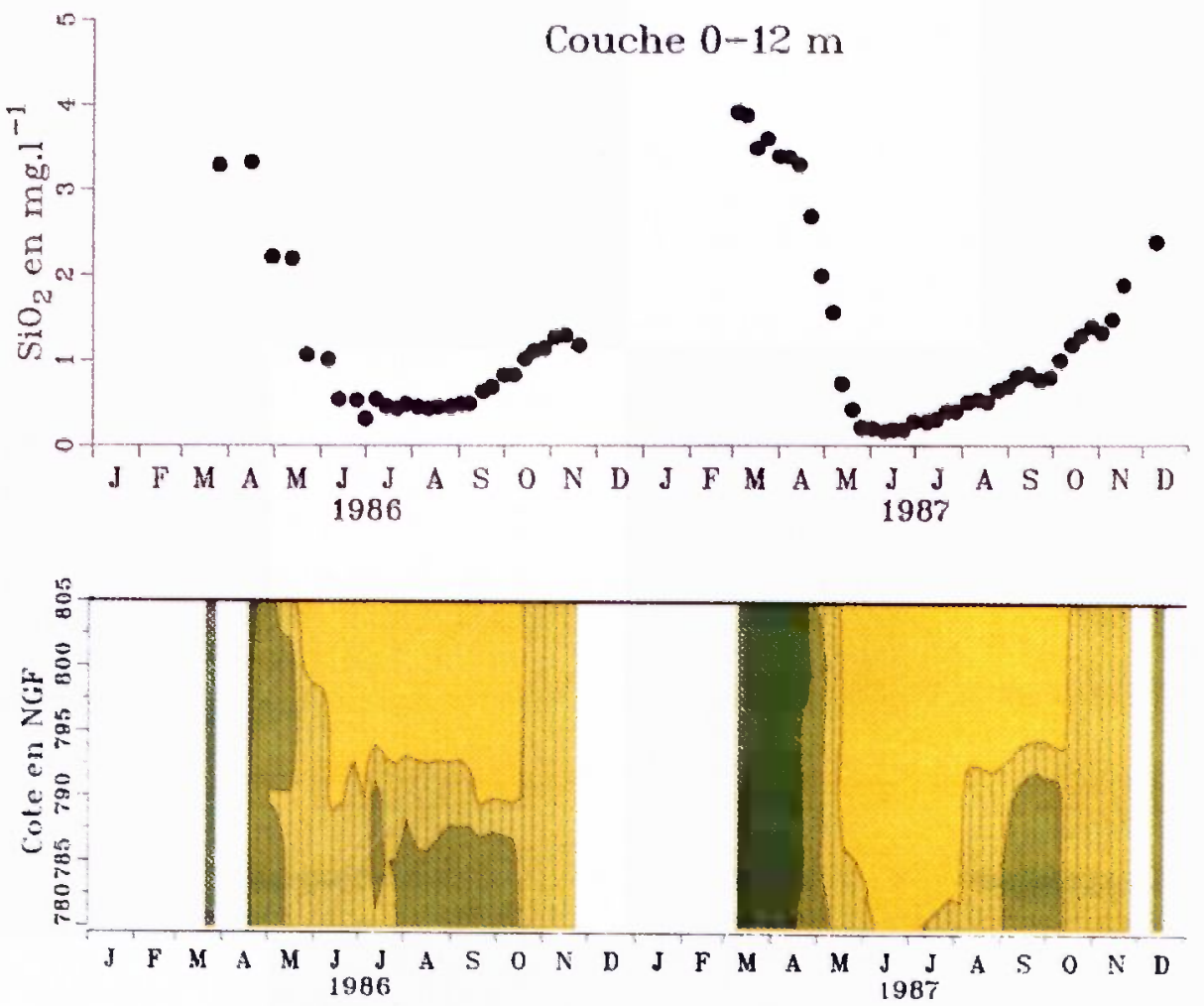

0.0

$\mathrm{SiO}_{2}$ en $\mathrm{mg.l}^{-i^{3.0}}$

$+0$

5.0

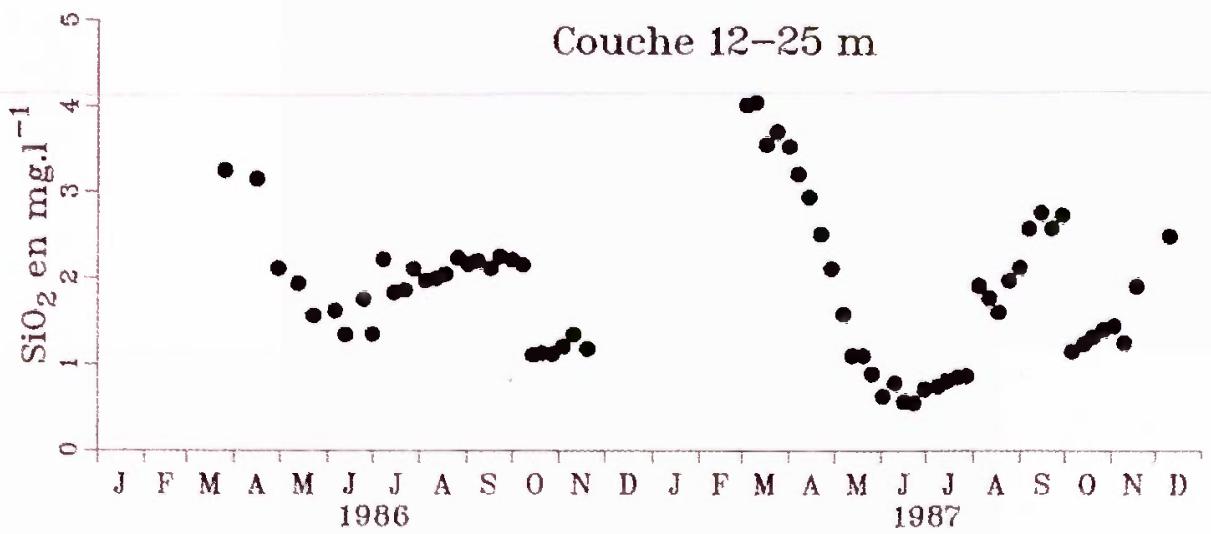

Fig. 10. - Evolution saisonnière des concentrations en silice $\left(\mathrm{SiO}_{2}\right)$ dans la colonne d'eau du lac de Pareloup en 1986 et 1987 : concentration moyenne dans les couches 0-12 m (en haut), 12-25 m (en bas) et courbes d'isoconcentration (au centre).

Fig. 10. - Seasonal evolution of silica $\left(\mathrm{SiO}_{2}\right)$ concentrations in the water column at Pareloup in 1986 and 1987: mean concentration in layers 0-12 m (top) and 12-25 m (bottom) and depth-time distribution (center). 
La production primaire brute printanière (de mars à juin), calculée d'après le modèle, est de $23 \mathrm{~g}$ de $\mathrm{C} \cdot \mathrm{m}^{-2}$ en 1986 et de $24 \mathrm{~g}$ en 1987. En tenant compte de la respiration, on obtient une production nette de $15 \mathrm{~g}$ en 1986 et de $15,6 \mathrm{~g}$ en 1987. La silice représente $45 \%$ du poids sec chez Asterionella formosa (in Reynolds, 1984). On peut estimer qu'un atome de silice est utilisé pour trois atomes de carbone de la cellule. La mobilisation printanière de silice est donc de $416 \mathrm{mM} \cdot \mathrm{m}^{-2}$ en 1986 et de $433 \mathrm{mM} \cdot \mathrm{m}^{-2}$ en 1987. Cette estimation ne représente que les deux tiers de la disparition de silice, ce qui sug- gère une sous-estimation de la production nette des diatomées par le modèle de photosynthèse.

\section{Les échanges sédiments-eau}

On peut aussi essayer d'évaluer l'importance du flux originaire des sédiments dans le renouvellement de la silice du lac. Ce flux est proportionnel à la désaturation de la colonne d'eau et à la nature de la source (minéralogie et surface de contact).

Les mesures réalisées en été, avec agitation, dans les cloches benthiques sont représentées sur la figure 11. Elles montrent que la saturation est

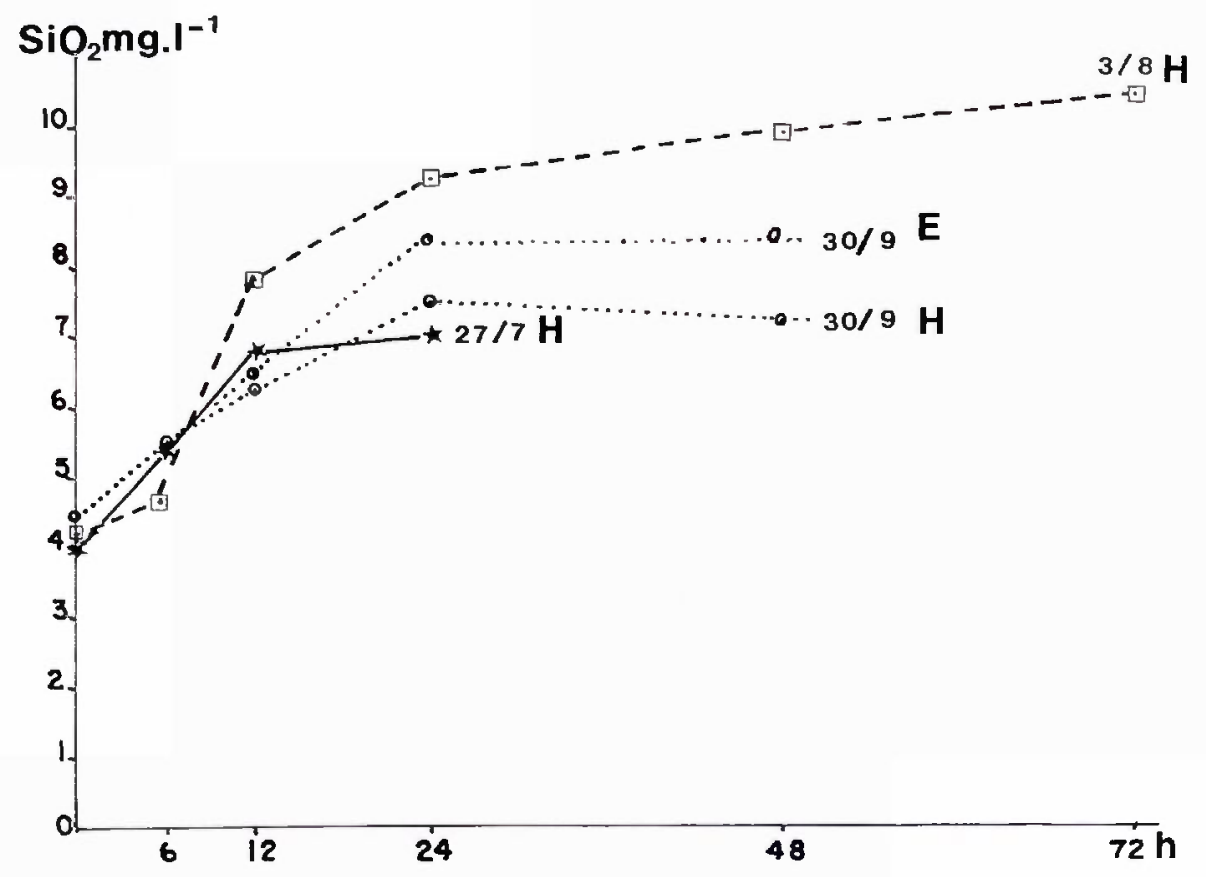

Fig. 11. - Evolution de la concentration en silice $\left(\mathrm{SiO}_{2}\right)$ dans les enceintes agitées au cours de l'été 1988. E : épilimnion. H: hypolimnion.

Fig. 11. - Evolution of the silica $\left(\mathrm{SiO}_{2}\right)$ concentration in the stirred chambers in summer 1988: $\mathrm{E}$ : epilimnion; $\mathrm{H}$ : hypolimnion. 
atteinte pour des valeurs de l'ordre de $120 \mu \mathrm{M} \cdot 1^{-1}$ en zone hypolimnique (température $=12^{\circ} \mathrm{C}$ ) ou $140 \mu \mathrm{M} \cdot \mathrm{I}^{-1}$ dans la zone épilimnique (température $>20^{\circ} \mathrm{C}$ ). Exceptionnellement, on dépasse $10500 \mu \mathrm{g} \cdot \mathrm{I}^{-1}\left(175 \mu \mathrm{M} \cdot \mathrm{I}^{-1}\right)$, à une température de $12^{\circ} \mathrm{C}$ environ. Ces valeurs, supérieures à la saturation par l'illite, indiquent une influence de la silice biogénique des frustules de Diatomées. Sans agitation, les équilibres sont atteints beaucoup plus lentement (fig. 12). Dans la zone épilimnique, les concentrations sont initialement plus faibles et atteignent $3300 \mu \mathrm{g} \cdot \mathrm{l}^{-1}\left(55 \mu \mathrm{M} \cdot \mathrm{I}^{-1}\right)$ seulement au bout de six jours.

Les mesures de flux effectuées sur les droites initiales des mesures en enceintes non agitées varient de 0,076 à $0,46 \mathrm{~g} \cdot \mathrm{m}^{-2} \cdot \mathrm{j}^{-1}$; elles sont $\mathrm{du}$ même ordre de grandeur que celles mesurées dans le lac Michigan (Conley et Schelske, 1989) ou sur les sédiments marins (Andrews et Hargrave, 1984) Ces flux sont à l'origine de la légère remontée des concentrations dans l'hypolimnion pendant la période de stratification, en l'absence d'apports de Bage.

\section{L'AZOTE}

Seuls les nitrates qui représentent plus de $85 \%$ des stocks de $\mathrm{N}$ ont été dosés de manière régulière.

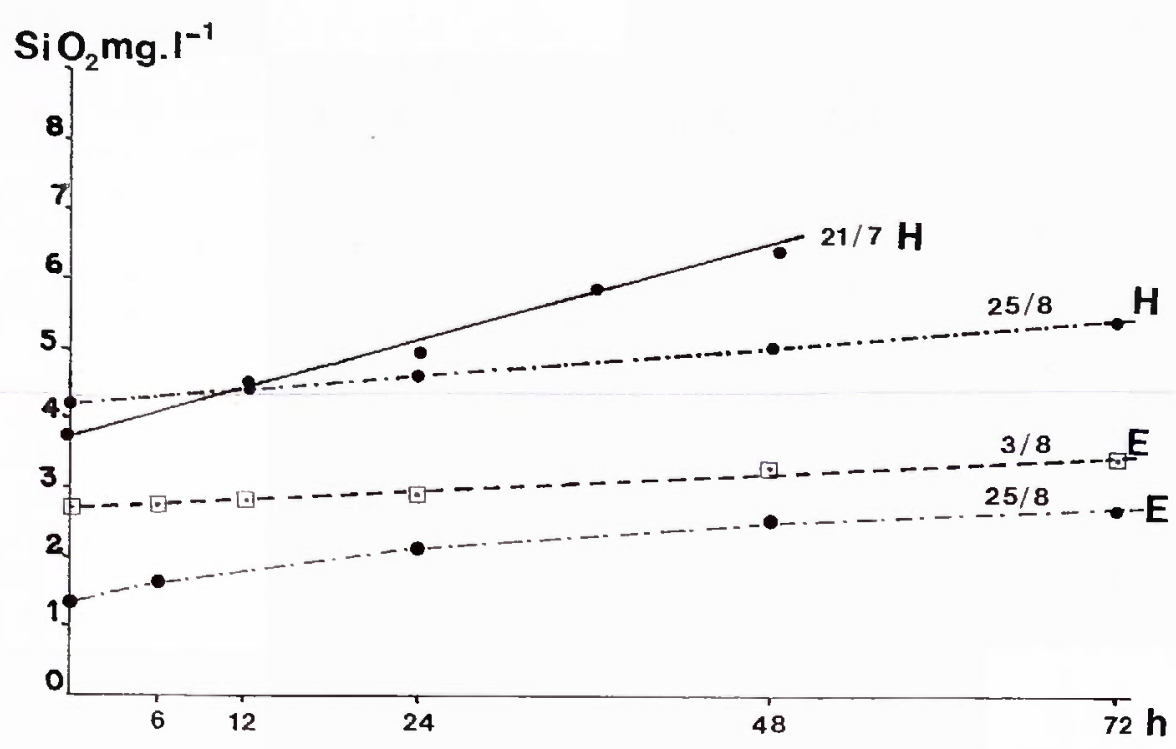

Fig. 12. - Evolution de la concentration en silice $\left(\mathrm{SiO}_{2}\right)$ dans les enceintes non agitées au cours de l'été 1988. $E$ : épilimnion. $H$ : hypolimnion.

Fig. 12. - Evolution of the silica $\left(\mathrm{SiO}_{2}\right)$ concentration in the unstirred chambers in summer 1988: $\mathrm{E}$ : epilimnion; H: hypolimnion. 


\section{Évolution des nitrates dans la co- lonne d'eau}

La concentration en nitrates est peu variable tout au long de l'année: 0,9 à $2 \mathrm{mg} \mathrm{N}-\mathrm{NO}_{3} \cdot \mathrm{I}^{-1}$ (fig. 13). Le maximum se situe au mois d'avril, au moment du lessivage des terres par les pluies, alors que la végétation n'est pas entièrement développée.

Un minimum s'observe au fond du lac à la fin de l'été 1987, en liaison avec la désoxygénation prononcée de l'hypolimnion, favorisant la dénitrification au niveau des sédiments.

Le rapport N/P est généralement bien supérieur à $10 \mathrm{ce}$ qui signifie que l'azote ne limite pas la production primaire.

\section{Nitrification - dénitrification au ni- veau des sédiments}

Le bilan a été évalué ponctuellement au moyen de cloches benthiques dans lesquelles la concentration en nitrates a été suivie en fonction du temps (fig. 14).

Les sédiments du lac se comportent toujours en puits, quelle que soit la zone envisagée. La consommation de nitrates augmente au cours de l'été (tableau II), probablement en raison de la désoxygénation (hypolimnion), de l'augmentation de température ou de l'apport de matière organique par sédimentation (épilimnion).

Les plus faibles valeurs de consommation mesurées, extrapolées à l'année, sont bien supérieures aux apports des précipitations. Le lac se comporte en système épurateur visà-vis de l'azote, la matière organique nécessaire à la dénitrification étant fournie par la photosynthèse algale.

\section{Ammonisation au niveau des sé- diments}

La production d'ammonium apparaît constante au niveau de l'hypolimnion, bien que très variable (fig. 15). Elle est négligeable au niveau de l'épilimnion, à l'exception de la fin septembre, en relation avec la déstratification et le dépôt de sédiments issus de l'hypolimnion par remise en suspension.

\section{L'OXYGÈNE}

L'oxygène est évidemment nécessaire à la vie piscicole mais aussi à la qualité de l'eau. En son absence, des respirations anaérobies se développent pouvant avoir un effet favorable (dénitrification) mais, le plus souvent, un effet défavorable : mise en solution de métaux réduits (manganèse, fer, métaux lourds) et du phosphore qui leur était lié, production d'hydrogène sulfuré $\left(\mathrm{H}_{2} \mathrm{~S}\right)$ et de méthane $\left(\mathrm{CH}_{4}\right)$.

En période de mélange, les eaux sont normalement saturées en oxygène, mais en période de stratification l'hypolimnion s'appauvrit progressivement car les transferts diffusifs d' $\mathrm{O}_{2}$ sont généralement inférieurs à $15 \%$ du déficit (Cornett et Rigler, 1987).

L'importance de la désaturation en oxygène de l'hypolimnion d'un lac semble liée au type trophique de ce lac, c'est à dire à sa productivité (Hutchinson, 1938). 

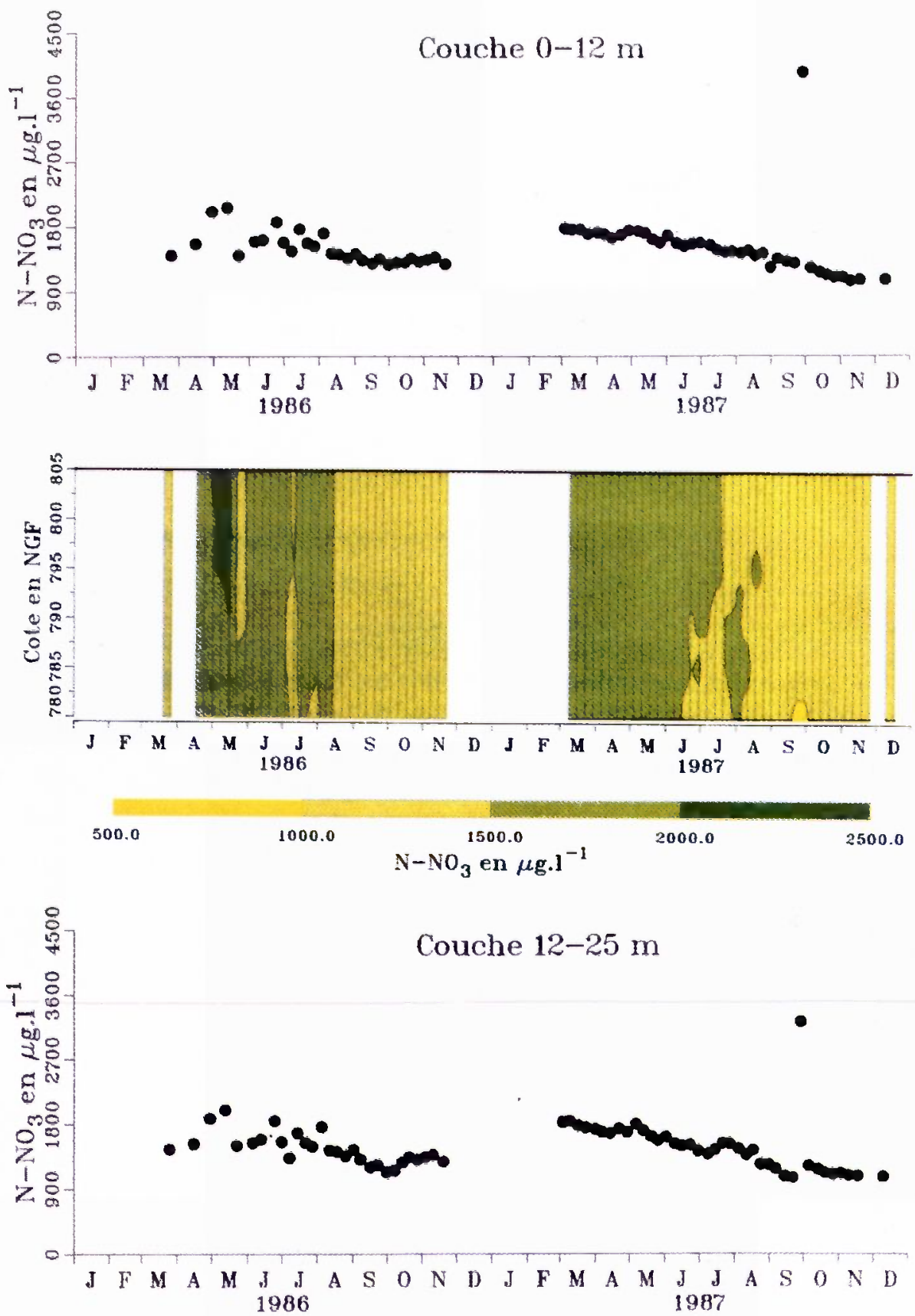

Fig. 13. - Evolution saisonnière de la concentration en nitrate dans la colonne d'eau du lac de Pareloup au cours des années 1986 et 1987 : concentration moyenne dans les couches 0-12 m (en haut), 12-25 m (en bas) et courbes d'isoconcentration (au centre).

Fig. 13. - Seasonal evolution of the nitrate concentration in the water column at Pareloup in 1986 and 1987: mean concentration in layers 0-12 $\mathrm{m}$ (top) and 12-25 m (bottom) and depth-time distribution (center). 

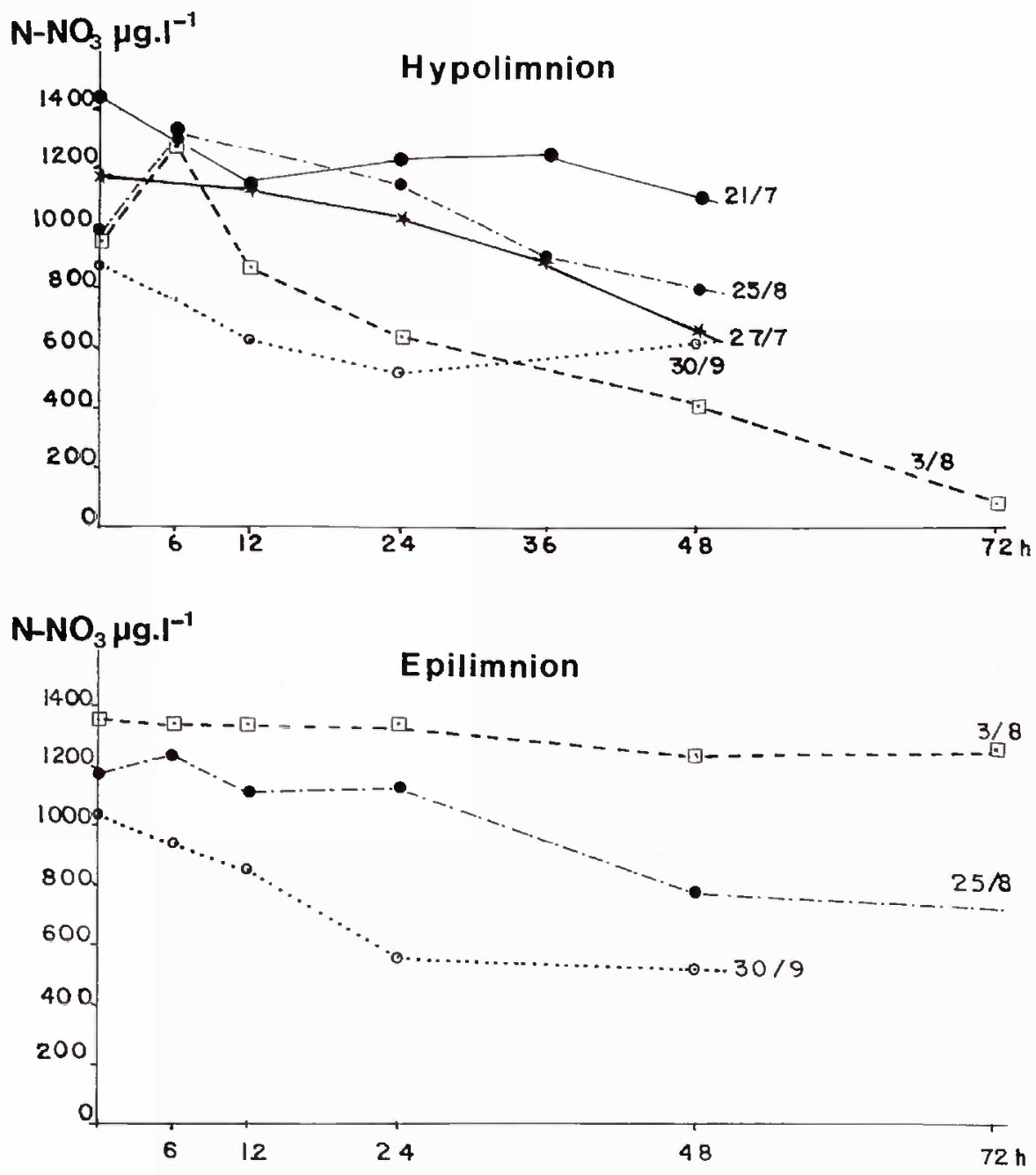

Fig. 14. - Consommation de nitrate dans les chambres benthiques au cours de l'été 1988. Fig. 14. - Nitrate consumption in the benthic chambers in summer 1988.

Tableau II. - Consommation de nitrate par les sédiments du lac de Pareloup au cours de l'été $1987\left(\mathrm{mg} \mathrm{N}-\mathrm{NO}_{3} \cdot \mathrm{m}^{-2} \cdot \mathrm{j}^{-1}\right)$.

Table Il. - Nitrate consumption by sediments in Pareloup in summer $1987\left(\mathrm{mg} \mathrm{N}-\mathrm{NO}_{3} \cdot \mathrm{m}^{-2} \cdot \mathrm{d}^{-1}\right)$.

\begin{tabular}{|c|c|c|}
\hline Dates & Hypolimnion & Epilimnion \\
\hline $27 / 07$ & 64 & - \\
$3 / 08$ & 109 & 20 \\
$25 / 08$ & 128 & 66 \\
$30 / 09$ & 140 & 159 \\
\hline
\end{tabular}



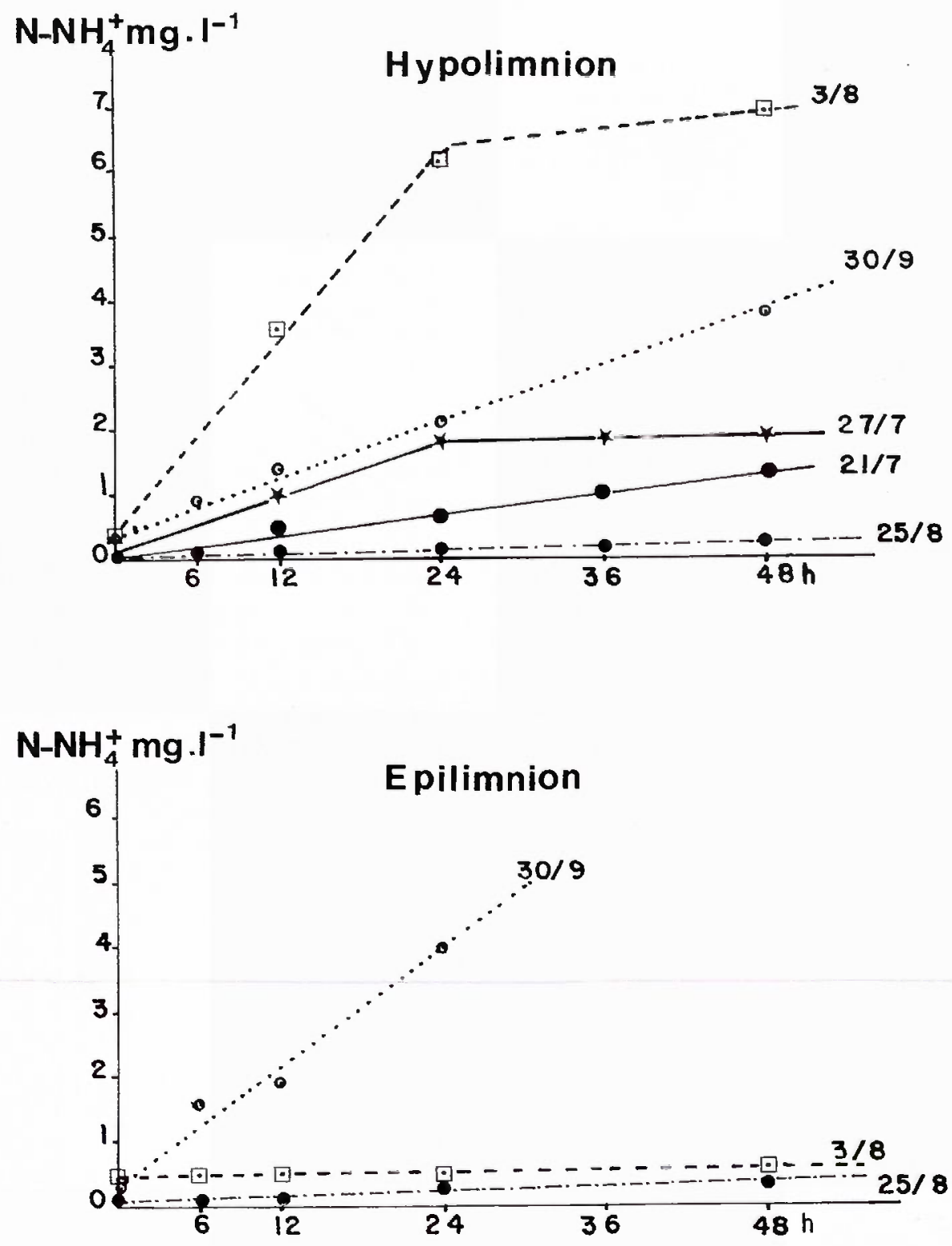

Fig. 15. - Production d'ammoniaque par les sédiments du lac de Pareloup au cours de l'été 1988. Fig. 15. - Ammonia production by sediments in Pareloup in summer 1988. 


\section{Évolution de l'oxygène dans la colonne d'eau}

La concentration en oxygène dissous dans la colonne d'eau au cours des années 1986 et 1987 semble suivre une évolution classique : après l'établissement de la stratification estivale, l'hypolimnion se désature progressivement alors que l'épilimnion reste saturé ou sursaturé en fonction de l'activité photosynthétique (fig. 16). Cependant, une étude plus approfondie des évènements contrôlant la concentration en oxygène de l'eau montre que les explications ne sont pas aussi simples.

Hutchinson $(1938,1957)$ introduisit la notion de vitesse de consommation d'oxygène hypolimnique par unité de surface et par jour (AHODR ou AHOD), afin de supprimer l'incidence de la morphométrie du lac sur l'évolution de l'oxygène hypolimnique; mais d'autres facteurs (température) agissent sur cette évolution. De nombreux auteurs ont établi des modèles empiriques pour prédire le niveau de la désaturation atteint par le lac en fonction de ces facteurs (Cornett et Rigler, 1979, 1980; Charlton, 1980; Vollenweider et Janus, 1982). Ces modèles ne sont pas applicables au lac de Pareloup car, comme nous le verrons, l'oxygénation de l'hypolimnion est tributaire des apports du réservoir de Bage.

L'évaluation de l'AHOD a été effectuée par la méthode de Lasenby (1975). La masse d'oxygène contenue dans l'hypolimnion à un instant donné est calculée en faisant la somme des masses caculées dans des tranches volumiques (volume $\times$ concentration). En divisant cette masse par la surface à la profondeur de référence (oxycline), on obtient la masse surfaciale de l'hypolimnion. La pente de la droite de régression de ces masses établie en fonction du temps (en juillet-août, alors que l'oxygène n'est pas limitant) donne la vitesse moyenne d'épuisement de l'oxygène dans l'hypolimnion ou AHOD (exprimée en $\mathrm{g} \cdot \mathrm{m}^{-2} \cdot \mathrm{j}^{-1}$ ). Des calculs analogues ont été réalisés pour la surface du lac et la zone profonde $(-16 \mathrm{~m})$ afin de préciser certains phénomènes.

Les résultats (fig. 17) montrent que ce calcul fournit une AHOD dépendant largement des apports de Bage dont l'eau désoxygénée est injectée dans l'hypolimnion. Si le lac n'est pas encore stratifié, c'est toute la colonne d'eau qui est concernée. Par exemple, entre le 5 et le 12 juillet 1986, la désoxygénation atteint $1,641 \mathrm{~g} \cdot \mathrm{m}^{-2} \cdot \mathrm{j}^{-1}$; entre le 2 et le 10 juin 1987 $1,746 \mathrm{~g} \cdot \mathrm{m}^{-2} \cdot \mathrm{j}^{-1}$, ce qui correspond à la consommation d'un lac hypereutrophe. Les effets se font sentir longtemps puisque la valeur minimale obtenue entre le 19 juillet et le 16 août 1986 est encore de $0,953 \mathrm{~g} \cdot \mathrm{m}^{-2} . \mathrm{j}^{-1}$, ce qui correspond à un lac nettement eutrophe selon Hutchinson (1957). En 1987, la valeur minimale est de $0,493 \mathrm{~g} \cdot \mathrm{m}^{-2} \cdot \mathrm{j}^{-1}$, caractéristique du statut de lac mésotrophe de Pareloup (mesure entre le 30 juin et le 22 juillet). Ensuite, les apports de l'eau de Bage, pompée sans arrêt au cours du mois de septembre, "eutrophisent" l'hypolimnion dont l'AHOD s'annule 

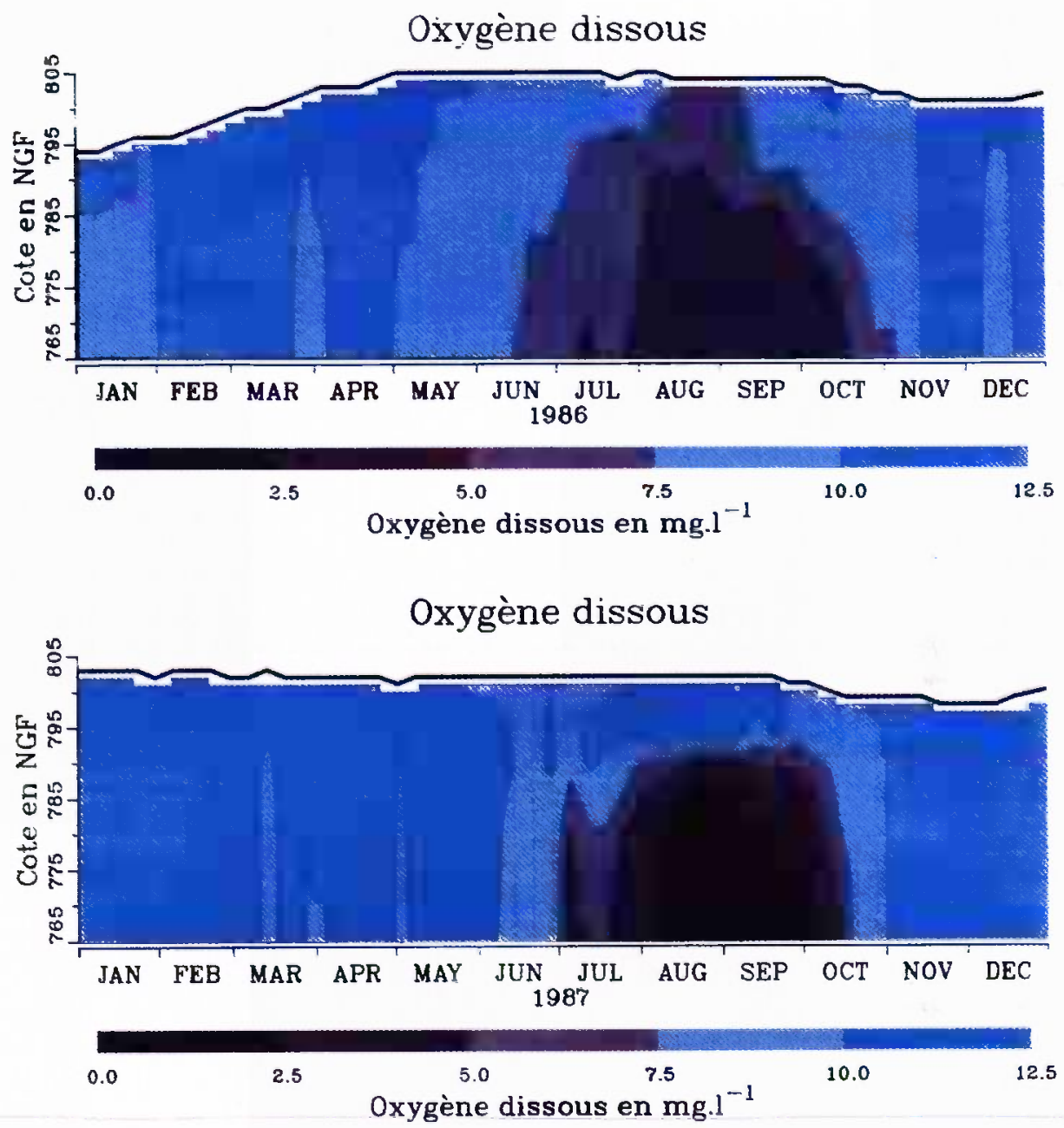

Fig. 16. - Evolution saisonnière des concentrations en oxygène dans la colonne d'eau du lac de Pareloup en 1986 et 1987.

Fig. 16. - Seasonal evolution of oxygen concentrations in the water column at Pareloup in 1986 and 1987.

par manque d'oxygène. La désoxygénation de l'hypolimnion s'accompagne d'une érosion de la thermocline de sorte qu'au niveau de l'hypolimnion tout entier et plus encore de la colonne d'eau le bilan n'est pas négatif (fig. 17). Lorsque les températures sont peu différentes, la thermocline disparaît alors qu'une pseudo-oxycline persiste (fig. 18). Cette situation paradoxale peut s'expliquer si l'on admet que le gradient de température ne dé- 

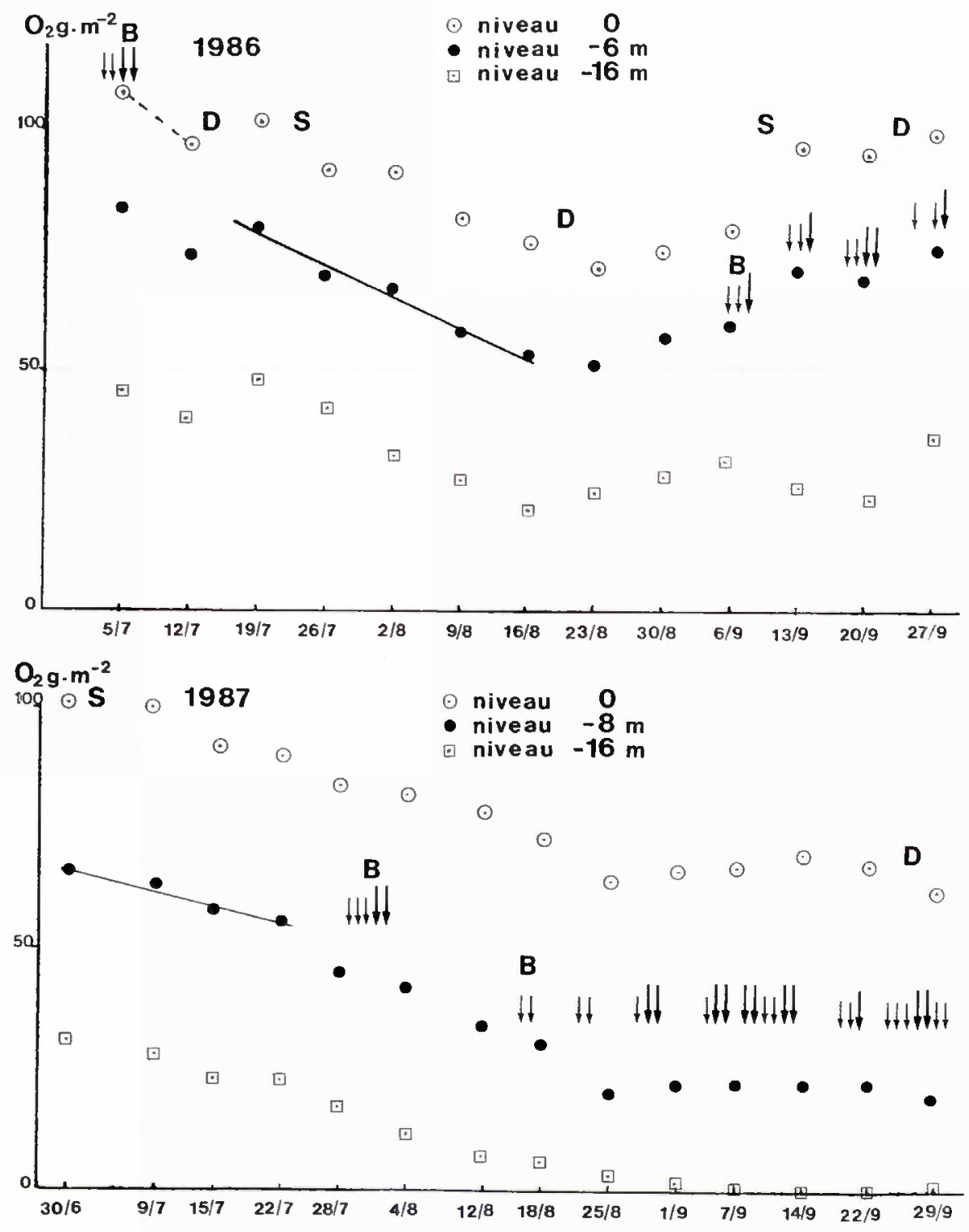

Fig. 17. - Evolution des masses moyennes d'oxygène par unité de surface $\left(\mathrm{g} \cdot \mathrm{m}^{-2}\right)$ sous différentes profondeurs au cours des étés 1986 et 1987. Les mesures de 1986 ont été prises à proximité du barrage; celles de 1987 depuis la plateforme EDF. Les évènements sont indiqués : B apports des réservoirs de Bage; la taille des flèches est grossièrement proportionnelle au débit (1 à $5 \mathrm{~m}^{3} \cdot \mathrm{s}^{-1}$; 5 à $\left.10 \mathrm{~m}^{3} \cdot \mathrm{s}^{-1}\right)$. S : stratification thermique. $\mathrm{D}$ : déstratification.

Fig. 17. - Evolution of mean oxygen masses per surface unit $\left(\mathrm{g} \cdot \mathrm{m}^{-2}\right)$ at different depths in summer 1986 and 1987. The 1986 measurements were taken near the dam: those of 1987, from the E.D.F. platform. Events are indicated: B: inflow from Bage reservoir; the size of the arrows is roughly proportional to the flow rate $\left(1\right.$ to $5 \mathrm{~m}^{3} \cdot \mathrm{s}^{-1} ; 5$ to $\left.10 \mathrm{~m}^{3} \cdot \mathrm{s}^{-1}\right)$. S: thermal stratification. D: destratification. 

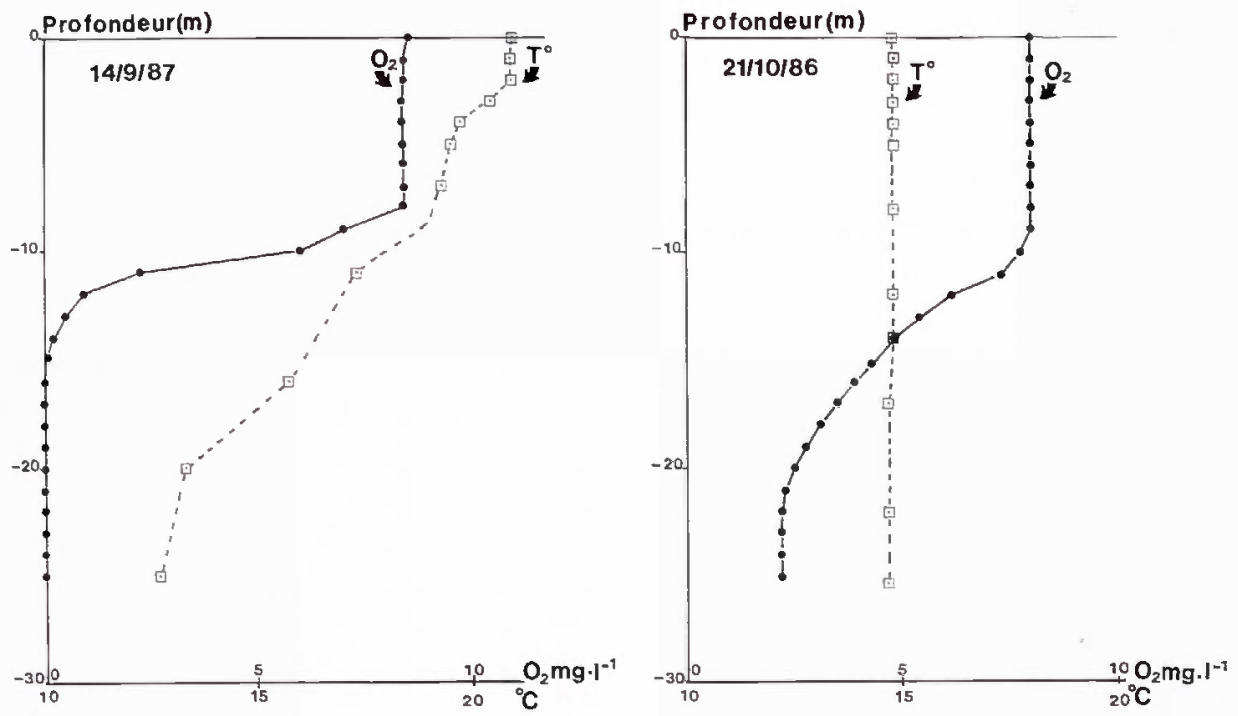

Fig. 18. - Profils verticaux de concentration en oxygène et de température montrant le rôle des apports du réservoir de Bage dans l'eutrophisation du lac de Pareloup: Formation d'une oxycline en l'absence de thermocline.

Fig. 18. - Vertical profiles for oxygen concentration and temperature showing the role of inflow from the Bage in eutrophication of Pareloup: formation of an oxycline in the absence of a thermocline.

pend que du mélange des eaux alors que le gradient d'oxygène dépend du mélange mais aussi du pouvoir réducteur de l'eau de Bage qui, apparemment, semble très élevé.

\section{Consommation d'oxygène au niveau des sédiments}

Des mesures de consommation d'oxygène par les sédiments ont été effectuées in situ, dans des cloches benthiques, au cours de l'été 1988 . Cette technique est évidemment plus lourde que les mesures effectuées en laboratoire sur des carottes de sédiment mais les résultats sont plus fiables (Seiki et al., 1989). Ces résultats montrent que la consommation d'oxygène peut atteindre $800 \mathrm{mg} \cdot \mathrm{m}^{-2} \cdot \mathrm{j}^{-1}$, ce qui correspond à un lac eutrophe. II apparaît que les sédiments peuvent assurer seuls la désoxygénation de l'hypolimnion lorsque l'eau de Bage n'intervient pas. En présence d'ap ports d'eau de Bage le rôle des sédiments est néammoins mis en évidence par la désoxygénation complète de la base de l'hypolimnion et la progression du phénomène vers le haut. Ce rôle primordial des sédiments a été déjà mis en évidence par Hargrave (1972).

Ces taux de consommation initiale élevés semblent correspondre à un compartiment labile, probablement al- 
gal. En effet, après 48 ou 72 heures, selon son importance, la consommation d'oxygène chute brutalement (fig. 19), diminuant d'un facteur 10. On peut aussi estimer que cette décroissance est liée à la chute de concentration en oxygène. En effet, en étudiant des microcosmes, Edwards et Rolley (1965) estiment que l'influence de la concentration en oxygène sur sa consommation suit une loi puissance :

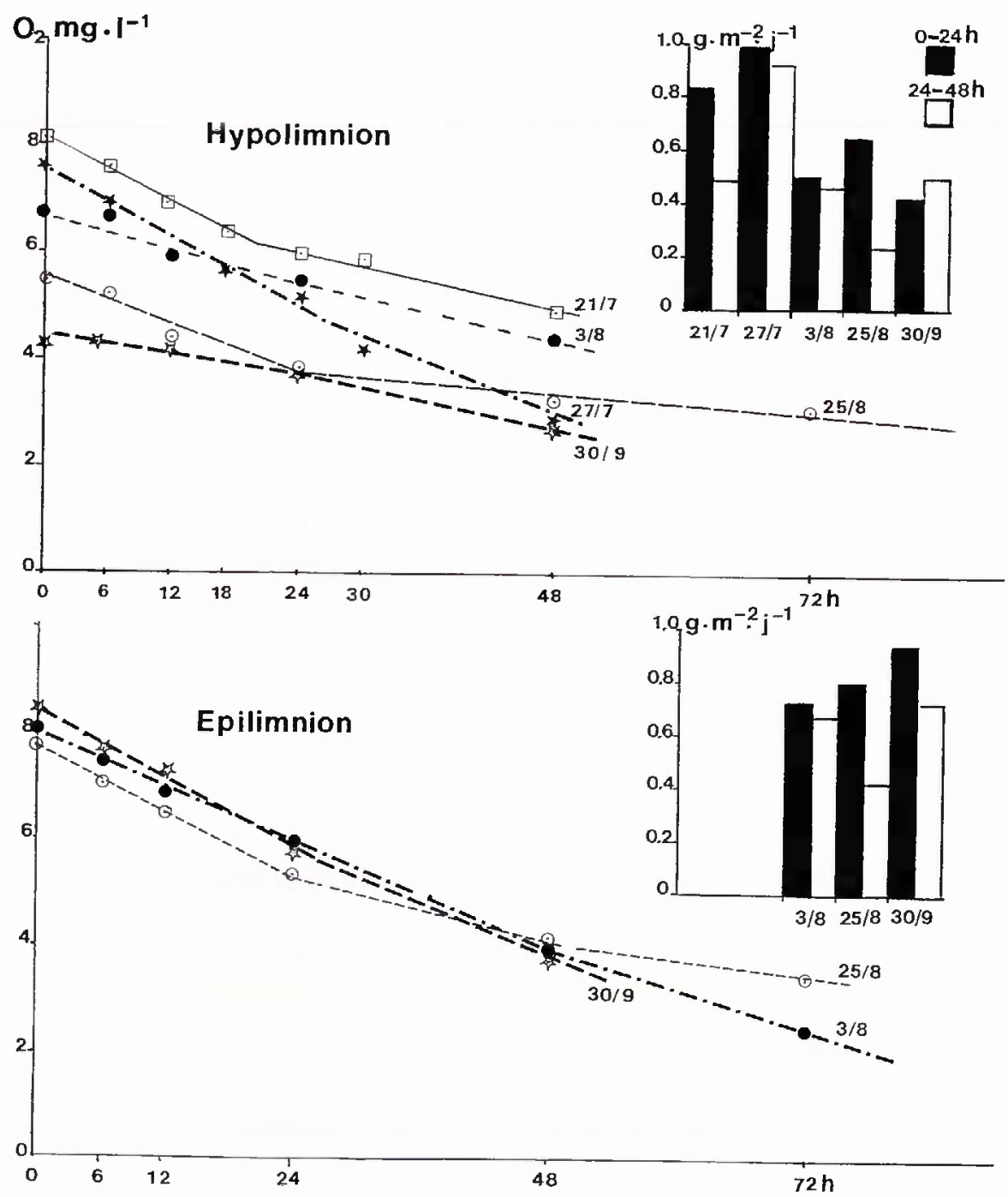

Fig. 19. - Consommation d'oxygène dans les cloches benthiques au cours de l'été 1988. Fig. 19. - Oxygen consumption in the benthic chambers in summer 1988. 


$$
y=a C^{b}
$$

avec $b$ \# 0,45

Toutefois la pente change nettement après 48 ou 72 heures quelle que soit la teneur en oxygène et Fillos (1976) n'observe pas d'influence tant que la concentration $\mathrm{d}^{\prime} \mathrm{O}_{2}$ reste supérieure à $2 \mathrm{ppm}$. Cependant, la consommation diminue en septembre dans les sédiments hypolimniques, en liaison sans doute avec la forte baisse de concentration en oxygène.

\section{CONCLUSION}

La dynamique des éléments biogènes dans la retenue de Pareloup est fortement influencée par les apports d'eau provenant de la retenue de Pont de Salars et pompés dans le réservoir de Bage. Le rôle de ces apports est particulièrement mis en évidence par l'évolution des concentrations d'oxygène. Ces apports contribuent sans doute aussi au pouvoir réducteur élevé manifesté par les sédiments, pouvoir essentiellement lié à du matériel labile donc probablement algal. En période de stratification, seul l'hypolimnion est concerné par ces apports, (Salençon et Calmels, 1994) l'épilimnion conservant un caractère de lac méso-eutrophe. Cependant, lors de la déstratification automnale, les nutriments stockés viennent s'ajouter à ceux apportés directement.

L'appréciation de la contribution des apports de Bage demanderait une meilleure connaissance des lois de mélange de l'eau pompée avec celle de la retenue de Pareloup, particulièrement en période non stratifiée. II n'est pas impossible que le turbinage affecte de manière préférentielle les eaux ainsi introduites dans l'hypolimnion, réduisant d'autant les effets eutrophisants de ces apports.

\section{BIBLIOGRAPHIE}

Andrews D. et Hargrave B.T., 1984. Close interval sampling of interstitial silicate and porosity in marine sediments. Geochimica et Cosmochimica Acta. V. 48, pp. 711-722.

Berner R.A., 1980. Early Diagenesis. A theoretical Approach. Princeton Univ. Press. $241 \mathrm{p}$.

Charlton M.N., 1980. Hypolimnion Oxygen Consumption in Lakes: Discussion of Productivity and Morphometry Effects. Can. J. Fish. Aquat. Sci., 37, pp. 15311539.

Conley D.J. et Schelske C.L., 1989. Processes controlling the benthic regeneration and sedimentary accumulation of biogenic silica in Lake Michigan. Arch. Hydrobiol., 116, 1, pp. 23-43.

Conway H.L., Parker J.J., Yaguchi E.M. et Mellinger D.L., 1977. Biological utilization and regeneration of silicon in Lake Michigan. J. Fish. Res. Bd Can., 34, pp. 537-544.

Cornett R.J., et Rigler F.H., 1979. Hypolimnetic Oxygen Deficits: Their Prediction and Interpretation. Science 205, pp. 580-582.

Cornett R.J., et Rigler F.H., 1980. The areal hypolimnetic deficit. An empirical test of the model. Limnol. Oceanogr. 25 , pp. $672-680$.

Cornett R.J., et Rigler F.H., 1987. Vertical transport of Oxygen into the Hypolimnion of Lakes. Can. J. Fish. Aquat. Sci., 4, pp. 852-858. 
Dalger D., 1982. Dosage du carbone et de l'azote organiques dans l'eau par chromatographie en phase gazeuse. Mise au point et applications de la méthode. Thèse $3^{\ominus}$ cycle. Université toulouse, $75 \mathrm{p}$.

Edwards R.W. et Rolley H.L.J., 1965. Oxygen consumption of river muds. $J$. Ecol., 53, pp. 1-19.

Elgawhary S. M. et Lindsay W. L., 1972. Solubility of Silica in soils. Soil Sci. Soc. Amer. Proc. 36, pp. 439-442.

Fillos J., 1976. Effects of sediments on the quality of the overlying water. In : "Interactions between sediments and fresh water". Dr W. Junk B.V. Publishers. The Hague 1977, pp. 266-271.

Goldman J.C., 1980. Physiological processes, nutrient availability and the concept of relative growth rate in phytoplankton ecology. In: "Primary production in the sea" (ed. P. Falkowski), Plenum, NY. pp. 179-194.

Hargrave B.T., 1972. Comparison of sediment oxygen uptake, hypolimnetic oxygen deficit and primary production in Lake Esrom, Danemark. Verh. Internat. Verein. Limnol., 18, pp. 134139.

Healey F.P. et Hendzel U., 1979. Indicators of phosphorus and nitrogen deficiency in five algae in culture. J. Fish. Res. Bd. Can. 36, pp. 1364-1369.

Hurd D.C., 1984. Inorganic Interactions of Silica-containing Minerals with Seawater. Marine Geology and Oceanography of Arabian Sea \& Coastal Pakistan. B.U. Haq \& J.D. Milliman (eds.). Van Nostrand Reinhold Co., N.Y., pp. 361-368.

Hutchinson G.E., 1938. On the relation between the oxygen deficit and the productivity and typology of lakes: Int. Rev. Hydrobiol., 36, pp. 336-355.

Hutchinson G.E., 1957. A treatise on limnology 1 . Wiley $1015 p$.
Lasenby D.C., 1975. Developments of oxygen deficits in 14 Southern Ontario lakes. Limnol. Oceanogr., 20, pp. 993998.

Lean D.R.S., 1973. Movement of phosphorus between its biologically important forms in lake water. J. Fish. Res. Bd. Can., 30 : pp. 1525-1536.

Motomizu S., Wakimoto T. et Tœi S., 1983. Spectrophotometric determination of phosphate in river waters with molybdate and malachite green. Analyst, 108 , pp. 361-367.

Paasche E., 1980. The Physiological Ecology of Phytoplancton. I. Morris edit. Blackwell Scientific Publications. pp. 259-284.

Reynolds C.S., 1984. The Ecology of Freshwater Phytoplancton. Cambridge University Press, $384 \mathrm{p}$.

Rigler F.H., 1968. Further observations inconsistent with the hypothesis that the molybdenum blue method measures orthophosphate in lake water. Limnol. Oceanogr., 13, pp. 7-13.

Salençon M.J. et Calmels P., 1994. Etude de la dynamique des masses d'eau du lac de Pareloup par traçages. Hydroécol. Appl. 6(1/2), pp. 19-58.

Schink D.R., Guinasso Jr. N.L., et Fanning K.A., 1975. Processes affecting the concentration of silica at the sedimentwater interface of the Atlantic Ocean. J. Geophys. Res. 80, 21, pp. 30133031.

Sivadier F., Thébault J.M. et Salençon M.J., 1994. Bilan du phosphore total dans la retenue de Pareloup. Hydroécol. Appl. 6(1/2), pp. 115-138.

Vollenweider R.A., et Janus L.L., 1982. Statistical models for predicting hypolimnetic depletion rates. Mem. Ist. Ital. Idrobiol., 40, pp. 1-24. 